\title{
AVERAGE DECAY OF THE FOURIER TRANSFORM OF MEASURES WITH APPLICATIONS
}

\author{
RENATO LUCÀ AND KEITH M. ROGERS
}

\begin{abstract}
We consider spherical averages of the Fourier transform of fractal measures and improve the lower bound on the rate of decay by taking advantage of multilinear estimates. Maximal estimates with respect to fractal measures are deduced for the Schrödinger and wave equations. This refines the almost everywhere convergence of the solution to its initial datum as time tends to zero. A consequence is that the solution to the wave equation cannot diverge on a $(d-1)$-dimensional manifold if the data belongs to the energy space $\dot{H}^{1}\left(\mathbb{R}^{d}\right) \times L^{2}\left(\mathbb{R}^{d}\right)$.
\end{abstract}

\section{INTRODUCTION}

Consider the Schrödinger equation, $i \partial_{t} u+\Delta u=0$, on $\mathbb{R}^{d}=\mathbb{R}^{n+1}$, with initial data $u(\cdot, 0)=u_{0}$ in $H^{s}$ defined by

$$
H^{s}=(1-\Delta)^{-s / 2} L^{2}:=\left\{G_{s} * f: f \in L^{2}\left(\mathbb{R}^{n}\right)\right\} .
$$

Here $G_{s}$ is the Bessel kernel defined as usual by $\widehat{G}_{s}=\left(1+|\cdot|^{2}\right)^{-s / 2}$, where ${ }^{-}$is the Fourier transform. In [17], Carleson considered the problem of identifying the exponents $s>0$ for which

$$
\lim _{t \rightarrow 0} u(x, t)=u_{0}(x), \quad \text { a.e. } \quad x \in \mathbb{R}^{n}, \quad \forall u_{0} \in H^{s},
$$

and proved that this property holds as long as $s \geq 1 / 4$ in the one-dimensional case. Dahlberg and Kenig [20] then showed that (1.1) does not hold if $s<1 / 4$.

The higher dimensional case has since been studied by many authors; see for example [19, 14, 43, 50, 7, 38, 39, 48, 47, 25]. In two dimensions, Lee [33] used bilinear techniques to prove that (1.1) holds if $s>3 / 8$. In higher dimensions, Bourgain took advantage of multilinear restriction estimates to prove that

$$
s>\frac{1}{2}-\frac{1}{4 n}
$$

is sufficient. Bourgain also improved the necessary condition of Dahlberg and Kenig when $n \geq 5$, showing that $s \geq 1 / 2-1 / n$ is necessary for (1.1) to hold. We improve his condition in the following theorem.

Theorem 1.1. If (1.1) holds, then

$$
s \geq \frac{1}{2}-\frac{1}{n+2} .
$$

This also improves the necessary condition of Dahlberg and Kenig when $n \geq 3$.

Key words and phrases. Hausdorff dimension, Fourier transform, Schrödinger equation.

Mathematics Subject Classification. Primary 42B37; Secondary 28A75.

Partially supported by the ERC grant 277778 and the MINECO grants MTM2013-41780-P, SEV-2015-0554 and MTM2017-85934-C3-1-P (Spain). The first author has also been partially supported by FIRB 2012 "Dispersive dynamics: Fourier Analysis and Variational Methods" (Italy). 
Altogether we see that (1.1) holds uniformly with respect to $n$ if and only if $s \geq 1 / 2$. A natural refinement of the problem is to bound the size of the divergence sets

$$
\mathcal{D}\left(u_{0}\right):=\left\{x \in \mathbb{R}^{n}: \lim _{t \rightarrow 0} u(x, t) \neq u_{0}(x)\right\},
$$

and in particular we consider

$$
\alpha_{n}(s):=\sup _{u_{0} \in H^{s}} \operatorname{dim}_{H}\left(\mathcal{D}\left(u_{0}\right)\right)
$$

where $\operatorname{dim}_{H}$ denotes the Hausdorff dimension. A completely satisfactory theory has already been developed in the one-dimensional case; see [2], [6], or [15]. Indeed

$$
\alpha_{n}(s) \leq n-2 s, \text { if } \quad \frac{n}{4} \leq s \leq \frac{n}{2},
$$

and this bound is sharp in the sense that initial data in $H^{s}$ can be singular on $\alpha$-dimensional sets when $\alpha<n-2 s$; see [53]. On the other hand, the solution is continuous (and so $\alpha_{n}(s)=0$ ) when $s>n / 2$, and the example of Dahlberg and Kenig tells us that $\alpha_{n}(s)=n$ when $s<1 / 4$. Altogether, the whole range is covered when $n=1$, and we note that $\alpha_{1}$ is discontinuous at $s=1 / 4$. These results and a more gentle introduction to the problem can also be found in [37, Chapter 17].

Here we improve the best known upper bounds for $\alpha_{n}(s)$ in the remaining range of interest, when $s<n / 4$, in higher dimensions. In particular, we prove the following theorem that refines the almost everywhere convergence due to Bourgain. At the same time, we improve the bound $\alpha_{n}(s) \leq n+1-2 s$ due to Sjögren and Sjölin [42] and the bound $\alpha_{n}(s) \leq \frac{n+3}{n+1}(n-2 s)$ due to Barceló, Bennett, Carbery and the second author [2].

Theorem 1.2. Let $n \geq 2$. Then

$$
\alpha_{n}(s) \leq \begin{cases}n+1-\left(2+\frac{2}{2 n-1}\right) s, & \frac{1}{2}-\frac{1}{4 n}<s \leq 1-\frac{3}{2(n+1)}, \\ n+1-\frac{1}{n+1}-2 s, & 1-\frac{3}{2(n+1)} \leq s<\frac{n}{4} .\end{cases}
$$

This will be a consequence of a maximal estimate (see Theorem 8.2) that holds uniformly with respect to fractal measures in the following class. To avoid repetition, we include positivity and a support condition inside the definition of ' $\alpha$ dimensional'.

Definition 1.3. Let $0<\alpha \leq d$. We say that $\mu$ is (at least) $\alpha$-dimensional if it is a positive Borel measure, supported in the unit ball $B(0,1)$, and satisfies

$$
c_{\alpha}(\mu):=\sup _{\substack{x \in \mathbb{R}^{d} \\ r>0}} \frac{\mu(B(x, r))}{r^{\alpha}}<\infty .
$$

The Fourier transform of such a measure need not decay in every direction (for example the Fourier transform of a piece of the surface measure on a hyperplane does not decay in the normal direction), however it must decay on average. As the class contains measures that are supported on $\alpha$-dimensional sets, the uncertainty principle suggests that there should be less decay for smaller values of $\alpha$. Let $\beta_{d}(\alpha)$ denote the supremum of the numbers $\beta$ for which ${ }^{1}$

$$
\|\widehat{\mu}(R \cdot)\|_{L^{2}\left(\mathbb{S}^{d-1}\right)}^{2} \lesssim c_{\alpha}(\mu)\|\mu\| R^{-\beta}
$$

\footnotetext{
${ }^{1}$ We write $A \lesssim B$ if $A \leq C B$ for some constant $C>0$ that only depends on the dimension $d$ and/or a small parameter $\varepsilon$, in this case $\varepsilon=\beta_{d}(\alpha)-\beta$. If the constant depends on anything else, say a power of $N$, we write $A \lesssim_{N} B$. We also write $A \simeq B$ if $A \lesssim B$ and $B \lesssim A$.
} 
whenever $R>1$ and $\mu$ is $\alpha$-dimensional. The problem of identifying the precise value of $\beta_{d}(\alpha)$ was proposed by Mattila; see for example [36, pp. 42] or [37, Chapter 15]. In two dimensions, the sharp decay rates are now known;

$$
\beta_{2}(\alpha)=\left\{\begin{array}{ccc}
\alpha, & \alpha \in(0,1 / 2], & \\
1 / 2, & \alpha \in[1 / 2,1], & \\
\alpha / 2, & \alpha \in[1,2], & \text { (Wattila [35]) }
\end{array}\right.
$$

The work of Wolff, later simplified by Erdoğan [21], improved upon a lower bound due to Bourgain [8] who was the first to bring Fourier restriction theory to bear on the problem. In higher dimensions, the best known lower bounds are

$$
\beta_{d}(\alpha) \geq\left\{\begin{array}{lll}
\alpha, & \alpha \in\left(0, \frac{d-1}{2}\right], & \\
\frac{d-1}{2}, & \alpha \in\left[\frac{d-1}{2}, \frac{d}{2}\right], & \\
\alpha-1+\frac{d+2-2 \alpha}{4}, & \alpha \in\left[\frac{d}{2}, \frac{d+2}{2}\right], & (\text { Erdoğan }[22,23]) \\
\alpha-1, & \alpha \in\left[\frac{d+2}{2}, d\right], & \text { (Sjölin [44]). }
\end{array}\right.
$$

On the other hand, by considering limits of simple measures supported on small sets; see for example [37, Chapter 15.2], it is easy to show that

$$
\beta_{d}(\alpha) \leq \begin{cases}\alpha, & \alpha \in(0, d-2], \\ \alpha-1+\frac{d-\alpha}{2}, & \alpha \in[d-2, d] .\end{cases}
$$

The second part, for $\alpha \geq d-2$, is given by what is sometimes known as the 'Knapp example'. We see that the difference between this upper bound and the best known lower bound is never more than one and the bounds coincide when $\alpha<\frac{d-1}{2}$ or $\alpha=d$. Worse counterexamples have been constructed for signed measures by Iosevich and Rudnev [31], or when the averages are taken over a piece of paraboloid rather than the sphere by Barceló, Bennett, Carbery, Ruiz and Vilela [3]. Indeed, there is an extensive literature regarding averages over different manifolds and other generalisations; see for example [12, 13, 27, 29, 28, 30, 45] and the references therein.

The following upper bound, due to Iosevich and Rudnev, follows from their conditional Theorem 3.1 in [32] after taking their convex body $K$ to be the unit ball, their $\gamma=0$, and combining with a number theoretic estimate that counts the number of times the square of a large integer can be represented as a sum of squares. The bound is not explicitly stated in [32], and has been overlooked in the recent literature, so we present it here.

Theorem 1.4. [32] Let $d \geq 5$. Then

$$
\beta_{d}(\alpha) \leq \alpha-1+\frac{2(d-\alpha)}{d} .
$$

Most of the article will be dedicated to proving the following lower bound, which improves the estimate of Sjölin for all $\alpha<d$ and the estimate of Erdoğan for ${ }^{2}$ $\alpha \geq d / 2+2 / 3+1 / d$.

Theorem 1.5. Let $d \geq 3$. Then

$$
\beta_{d}(\alpha) \geq \alpha-1+\frac{(d-\alpha)^{2}}{(d-1)(2 d-\alpha-1)} .
$$

\footnotetext{
$2_{\text {in fact in a very slightly larger range. }}$
} 
This is not enough to improve the state-of-the-art for the Falconer distance set conjecture (the argument of Mattila [35] combined with Theorem 1.5 implies that distance sets associated to $\alpha$-dimensional sets have positive Lebesgue measure whenever $\alpha>d / 2+5 / 12)$. On the other hand, the difference between the best known upper and lower bounds is now strictly less than $5 / 6$, from which we can deduce new information regarding the pointwise convergence of solutions to the wave equation.

Considering $\partial_{t t} v=\Delta v$ on $\mathbb{R}^{d+1}$, with $v(\cdot, 0)=v_{0}$ and $\partial_{t} v(\cdot, 0)=v_{1}$, we take initial data in the homogeneous space $\dot{H}^{s} \times \dot{H}^{s-1}$, where

$$
\dot{H}^{s}=(-\Delta)^{-s / 2} L^{2}:=\left\{I_{s} * f: f \in L^{2}\left(\mathbb{R}^{d}\right)\right\} .
$$

Here $I_{s}$ is the Riesz kernel defined by $\widehat{I}_{s}=|\cdot|^{-s}$. The almost everywhere convergence question was first considered by Cowling [19], who proved

$$
\lim _{t \rightarrow 0} v(x, t)=v_{0}(x), \quad \text { a.e. } \quad x \in \mathbb{R}^{d}, \quad \forall\left(v_{0}, v_{1}\right) \in \dot{H}^{s} \times \dot{H}^{s-1}
$$

as long as $s>1 / 2$. Walther [51] then proved that this is not true when $s \leq 1 / 2$, and so the Lebesgue measure question is completely solved for the wave equation. As before we write

$$
\mathcal{D}\left(v_{0}, v_{1}\right):=\left\{x \in \mathbb{R}^{d}: \lim _{t \rightarrow 0} v(x, t) \neq v_{0}(x)\right\},
$$

and consider the refined problem of providing upper bounds for

$$
\gamma_{d}(s):=\sup _{\left(v_{0}, v_{1}\right) \in \dot{H}^{s} \times \dot{H}^{s-1}} \operatorname{dim}_{H}\left(\mathcal{D}\left(v_{0}, v_{1}\right)\right) .
$$

Sharp estimates were proven in the two-dimensional case in [2], using the following proposition which forms the link with the decay estimate (1.2).

Proposition 1.6. Let $d \geq 2$ and $0<s<d / 2$. Then $\beta_{d}(\alpha)>d-2 s \Rightarrow \gamma_{d}(s) \leq \alpha$.

Estimates for the inhomogeneous spaces $H^{s}\left(\mathbb{R}^{d}\right)$ were proven in [2], which puts unnecessary restrictions on the data $v_{1}$, however we will see that the implication also holds in this slightly more general context. Using Sjölin's bound $\beta_{d}(\alpha) \geq \alpha-1$ they deduced that $\gamma_{d}(s) \leq d+1-2 s$, so a consequence of Theorem 1.5 is that $\gamma_{d}(1)<d-1$, ruling out divergence on spheres if the initial data belongs to the energy space $\dot{H}^{1}\left(\mathbb{R}^{d}\right) \times L^{2}\left(\mathbb{R}^{d}\right)$.

The exponent $\beta_{d}(\alpha)$ is also connected to dimension estimates for orthogonal projections; see for example the recent work of Oberlin-Oberlin [41]. For a related problem regarding Fourier convergence at the points where the function is zero, see [16] or [18] and the references therein.

Although Theorem 1.5 yields new bounds for the Schrödinger equation, via an appropriate version of Proposition 1.6, those presented in Theorem 1.2 follow by a more direct use of the techniques developed to prove Theorem 1.5. Compared to the cone, the paraboloid has an extra nonzero principal curvature, and so it is not always efficient to use Proposition 1.6 in that case. For this reason we have presented the results for the Schrödinger equation in $\mathbb{R}^{n+1}$ and the results for the wave equation in $\mathbb{R}^{d+1}$, where $d=n+1$, and this convention will be maintained throughout.

It seems unlikely that our lower bound for $\beta_{d}(\alpha)$ is best possible. Indeed, Iosevich and Rudnev [32] proved that $\beta_{d}(\alpha) \geq \alpha-\alpha / d$ when restricting attention to a class of well-distributed measures and conjectured that this should hold for the full class of $\alpha$-dimensional measures.

The key ingredient in the proofs of Theorems 1.2 and 1.5 will be the multilinear extension estimate due to Bennett, Carbery and Tao [5], which was first successfully employed to prove linear estimates by Bourgain and Guth [11]. We present 
the multilinear estimates in Section 4 and a decomposition due to Bourgain and Guth in Section 5. In Section 6 we prove Theorem 1.5 and in Section 8 we prove Theorem 1.2. In Section 7 we present the simple proof of Proposition 1.6, via polar coordinates. In the following section we prove Theorem 1.1 and in the third section we give a direct proof of Theorem 1.4. The principal novelties, if any, will be described near the beginning of each section.

\section{Proof of Theorem 1.1}

When the initial data $u_{0}$ is a Schwartz function, the solution $u$ to the Schrödinger equation can be written as

$$
u(x, t)=e^{i t \Delta} u_{0}(x):=\frac{1}{(2 \pi)^{n / 2}} \int_{\mathbb{R}^{n}} \widehat{u}_{0}(\xi) e^{i x \cdot \xi-i t|\xi|^{2}} d \xi,
$$

where $\widehat{u}_{0}$ denotes the Fourier transform of $u_{0}$. By the Nikišin-Stein maximal principle $[40,46]$, the almost everywhere convergence (1.1) implies a weak $L^{2}$-estimate for the maximal operator, which in turn implies the strong estimate (2.2) by interpolation with a trivial bound (see for example [2, Proof of Lemma C.1]). Thus Theorem 1.1 is a consequence of the following theorem.

Theorem 2.1. Let $n \geq 3$ and suppose that there is a constant $C_{s}$ such that

$$
\left\|\sup _{0<t<1}\left|e^{i t \Delta} f\right|\right\|_{L^{2}(B(0,1))} \leq C_{s}\|f\|_{H^{s}\left(\mathbb{R}^{n}\right)}
$$

whenever $f$ is a Schwartz function. Then $s \geq \frac{n}{2(n+2)}$.

The counterexample of Dahlberg and Kenig can be interpreted as a concentrated solution, or wave-packet, that travels over a large area, making the left-hand side of (2.2) large. On the other hand, Bourgain considered a sum of data, with different velocities, carefully chosen to create regions of constructive interference, recalling Young's double slit experiment. Again the regions of coherence travel over a large area, making the left-hand side of the maximal inequality large.

In the light of Bourgain's example, a physical interpretation of Carleson's problem could be to identify the lowest frequency at which an initial state can generate interference patterns, thus obscuring its original state. Inspired by this, we take a variant of the data considered in [3] for which the corresponding solution interferes with itself periodically in time. The difficulty of using their example directly in this context is that the constructive interference reoccurs in the same relatively small regions of space. In order to take advantage of the periodic coherence, we perturb the initial state so that the whole solution travels in a single direction. We then use an ergodicity argument to show that this direction can be taken so that the regions of constructive interference never reappear in exactly the same places, forcing the left-hand side of (2.2) to be large.

We first prove the ergodic lemma. We say that a set $E$ is $\delta$-dense in $F$ if for every point $x \in F$ there is a point $y \in E$ such that $|x-y| \leq \delta$. The following lemma is optimal in the sense that the statement fails for larger $\kappa$. To see this, we can place balls of radius $\varepsilon R^{-1}$ at the points of the set $E_{\theta}$ and assume that the balls are disjoint. Then the volume of such a set would be of the order $R^{1-(n+2) \kappa}$, a quantity that tends to zero as $R$ tends to infinity when $\kappa>\frac{1}{n+2}$.

Lemma 2.2. Let $n \geq 3$ and $0<\kappa<\frac{1}{n+2}$. Then, for all $\varepsilon>0$ and sufficiently large $R>1$, there exists $\theta \in \mathbb{S}^{n-1}$ such that

$$
E_{\theta}:=\bigcup_{t \in R^{2 \kappa-1} \mathbb{Z} \cap(0,1)}\left\{x \in R^{\kappa-1} \mathbb{Z}^{n}:|x| \leq 2\right\}+t \theta
$$

is $\varepsilon R^{-1}$-dense in $B(0,1 / 2)$. 
Proof. By rescaling, the statement of the lemma is equivalent to showing that

$$
\bigcup_{t \in R^{\kappa} \mathbb{Z} \cap\left(0, R^{1-\kappa}\right)}\left\{x \in \mathbb{Z}^{n}:|x| \leq 2 R^{1-\kappa}\right\}+t \theta
$$

is $\varepsilon R^{-\kappa}$-dense in $B\left(0, R^{1-\kappa} / 2\right)$ for a certain $\theta \in \mathbb{S}^{n-1}$. That is to say, for any $x \in B\left(0, R^{1-\kappa} / 2\right)$ there exists a $y_{x} \in \mathbb{Z}^{n} \cap B\left(0,2 R^{1-\kappa}\right)$ and $t_{x} \in R^{\kappa} \mathbb{Z} \cap\left(0, R^{1-\kappa}\right)$ such that

$$
\left|x-\left(y_{x}+t_{x} \theta\right)\right| \leq \varepsilon R^{-\kappa},
$$

for a certain fixed $\theta \in \mathbb{S}^{n-1}$, independent of $x$. By taking the quotient $\mathbb{R}^{n} / \mathbb{Z}^{n}=\mathbb{T}^{n}$, this would follow if for any $[x] \in \mathbb{T}^{n}$ there exists $t_{x} \in R^{\kappa} \mathbb{Z} \cap\left(0, R^{1-\kappa}\right)$ such that

$$
\left|[x]-\left[t_{x} \theta\right]\right| \leq \varepsilon R^{-\kappa} .
$$

To see this, assume (2.4) and cover $B\left(0, R^{1-\kappa}\right)$ with a family of disjoint copies of axis-parallel $\mathbb{T}^{n}$. Denote the copy that contains $x$ by $\mathbb{T}_{x}^{n}$, and let $z_{x}$ be the point in $\mathbb{T}_{x}^{n}$ such that $\left[z_{x}\right]=\left[t_{x} \theta\right]$. Then $y_{x}:=z_{x}-t_{x} \theta \in \mathbb{Z}^{n}$ and by construction

$$
\left|x-\left(y_{x}+t_{x} \theta\right)\right|=\left|[x]-\left[t_{x} \theta\right]\right| \leq \varepsilon R^{-\kappa} .
$$

Note that we also automatically have that

$$
\left|y_{x}\right| \leq|x|+\left|t_{x}\right|+\varepsilon R^{-\kappa} \leq \frac{1}{2} R^{1-\kappa}+R^{1-\kappa}+\varepsilon R^{-\kappa}<2 R^{1-\kappa},
$$

and so we recover all of the required properties. It seems likely that ergodic results, similar to (2.4), are well-known, however we prove this using Fourier series as in [9]. We write $x$ instead of $[x]$ from now on.

Let $\phi: \mathbb{T}^{n} \rightarrow\left[0,(2 / \varepsilon)^{n}\right)$ be smooth, supported in $B(0, \varepsilon / 2)$, such that $\int \phi=1$, and set

$$
\phi_{R}(x):=\phi\left(R^{\kappa} x\right) .
$$

If we could show that there exists $\theta \in \mathbb{S}^{n-1}$ such that for all $x \in \mathbb{T}^{n}$ there is a $t_{x} \in\left(R^{\kappa} \mathbb{Z}+\left[-\frac{\varepsilon}{2} R^{-\kappa}, \frac{\varepsilon}{2} R^{-\kappa}\right]\right) \cap\left(0, R^{1-\kappa}\right)$ satisfying

$$
\phi_{R}\left(x-t_{x} \theta\right)>0 \text {, }
$$

then (2.4) would follow. Let $\psi:(-\varepsilon / 2, \varepsilon / 2) \rightarrow[0,2 / \varepsilon)$ be a one-dimensional Schwartz function such that $\int \psi=1$, and define

$$
\eta_{R}(t):=R^{3 \kappa-1} \sum_{\substack{j \in \mathbb{Z} \\ 0<j<R^{1-2 \kappa}}} \psi\left(R^{\kappa}\left(t-R^{\kappa} j\right)\right) .
$$

Noting that $\eta_{R}$ is supported in $R^{\kappa} \mathbb{Z}+\left[-\frac{\varepsilon}{2} R^{-\kappa}, \frac{\varepsilon}{2} R^{-\kappa}\right]$, we will show that there exists $\theta \in \mathbb{S}^{n-1}$ such that, for all $x \in \mathbb{T}^{n}$,

$$
\int_{\mathbb{R}} \phi_{R}(x-t \theta) \eta_{R}(t) d t>0
$$

which implies (2.8). Expanding in Fourier series;

$$
\phi_{R}(x-t \theta)=\widehat{\phi_{R}}(0)+\sum_{\substack{k \in \mathbb{Z}^{n} \\ k \neq 0}} \widehat{\phi_{R}}(k) e^{2 \pi i x \cdot k} e^{-2 \pi i t \theta \cdot k}=: \widehat{\phi_{R}}(0)+\Phi(t, x, \theta),
$$

and noting that $\int_{\mathbb{R}} \eta_{R} \simeq 1$ and $\widehat{\phi_{R}}(0)=\int_{\mathbb{T}^{n}} \phi_{R} \simeq R^{-n \kappa}$, it would be sufficient to find $\theta \in \mathbb{S}^{n}$ such that

$$
\left|\int_{\mathbb{R}} \Phi(t, x, \theta) \eta_{R}(t) d t\right| \lesssim R^{-\gamma}, \quad \gamma>n \kappa
$$

whenever $x \in \mathbb{T}^{n}$. 
For the proof of (2.10), we note that

$$
\begin{aligned}
\left|\int_{\mathbb{R}} \Phi(t, x, \theta) \eta_{R}(t) d t\right| & \leq \sum_{\substack{k \in \mathbb{Z}^{n} \\
k \neq 0}}\left|\widehat{\phi_{R}}(k)\right|\left|\int_{\mathbb{R}} e^{-2 \pi i t \theta \cdot k} \eta_{R}(t) d t\right| \\
& =\sum_{\substack{k \in \mathbb{Z}^{n} \\
k \neq 0}}\left|\widehat{\phi_{R}}(k)\right|\left|\widehat{\eta_{R}}(\theta \cdot k)\right| \\
& \lesssim \sum_{\substack{k \in \mathbb{Z}^{n} \\
k \neq 0}} \frac{R^{-n \kappa}}{\left(1+R^{-\kappa}|k|\right)^{n+1}}\left|\widehat{\eta_{R}}(\theta \cdot k)\right|
\end{aligned}
$$

where the final inequality follows by integrating by parts in the formula for the Fourier coefficients. Noting that the right-hand side of (2.11) no longer depends on $x$, so in order to find a $\theta \in \mathbb{S}^{n-1}$ such that (2.10) holds for all $x \in \mathbb{T}^{n}$, it will suffice to prove that the the right-hand side of (2.11) is similarly bounded after averaging over the sphere. As

$$
\sum_{\substack{k \in \mathbb{Z}^{n} \\ k \neq 0}} \frac{R^{-n \kappa}}{\left(1+R^{-\kappa}|k|\right)^{n+1}} \lesssim \int_{\mathbb{R}^{n}} \frac{R^{-n \kappa}}{\left(1+R^{-\kappa}|k|\right)^{n+1}} d k \lesssim 1
$$

by Fubini's theorem, for this it would suffice to prove that

$$
\int_{\mathbb{S} n-1}\left|\widehat{\eta_{R}}(\theta \cdot k)\right| d \theta \lesssim R^{2 \kappa-1} \log R
$$

Here we use that $\kappa<\frac{1}{n+2}$ so that $1-2 \kappa>n \kappa$.

To see (2.12), we note that

$$
\begin{aligned}
\widehat{\eta_{R}}(t) & =R^{3 \kappa-1} \sum_{\substack{j \in \mathbb{Z} \\
0<j<R^{1-2 \kappa}}} \psi\left(R^{\kappa}\left(\cdot-R^{\kappa} j\right)\right)^{\wedge}(t) \\
& =R^{2 \kappa-1} \widehat{\psi}\left(R^{-\kappa} t\right) \sum_{\substack{j \in \mathbb{Z} \\
0<j<R^{1-2 \kappa}}} e^{-2 \pi i R^{\kappa} j t} \\
& =R^{2 \kappa-1} \widehat{\psi}\left(R^{-\kappa} t\right) \frac{e^{2 \pi i\left\lfloor R^{1-2 \kappa}\right\rfloor R^{\kappa} t}-e^{-2 \pi i R^{\kappa} t}}{e^{2 \pi i R^{\kappa} t}-1} .
\end{aligned}
$$

Now since $|\widehat{\psi}| \lesssim 1$ this yields

$$
\int_{\mathbb{S}^{n-1}}\left|\widehat{\eta_{R}}(\theta \cdot k)\right| d \theta \lesssim R^{2 \kappa-1} \int_{\mathbb{S}^{n-1}}\left|\frac{\sin \left(\pi N R^{\kappa} \theta \cdot k\right)}{\sin \left(\pi R^{\kappa} \theta \cdot k\right)}\right| d \theta,
$$

where $N=\left\lfloor R^{1-2 \kappa}\right\rfloor+1$. By the Funk-Hecke theorem (see for example [1, pp. 35-36]), we have that

$$
\begin{aligned}
\int_{\mathbb{S}^{n-1}}\left|\frac{\sin \left(\pi N R^{\kappa} \theta \cdot k\right)}{\sin \left(\pi R^{\kappa} \theta \cdot k\right)}\right| d \theta & =\left|\mathbb{S}^{n-2}\right| \int_{-1}^{1}\left|\frac{\sin \left(\pi N R^{\kappa}|k| t\right)}{\sin \left(\pi R^{\kappa}|k| t\right)}\right|\left(1-t^{2}\right)^{\frac{n-3}{2}} d t \\
& \leq \frac{\left|\mathbb{S}^{n-2}\right|}{|k| R^{\kappa}} \int_{-|k| R^{\kappa}}^{|k| R^{\kappa}}\left|\frac{\sin (\pi N t)}{\sin (\pi t)}\right| d t \\
& \lesssim \log N \lesssim \log R
\end{aligned}
$$

where the penultimate inequality is a well-known property of the Dirichlet kernel (see for example [26, pp. 182]). This completes the proof of (2.12) which completes the proof of the lemma. 
Proof of Theorem 2.1. Writing $t /(2 \pi R)$ in place of $t$, the maximal estimate $(2.2)$ implies that

$$
\left\|\sup _{0<t<1}\left|e^{i \frac{t}{2 \pi R} \Delta} f\right|\right\|_{L^{2}(B(0,1))} \lesssim R^{s}\|f\|_{2}
$$

whenever supp $\widehat{f} \subset B(0,2 R)$ and $R>1$. In fact this is equivalent to (2.2); see [33] or [34], so we have not thrown anything away here. Thus it suffices to prove that $s \geq \frac{n}{2(n+2)}$ is necessary for (2.14) to hold.

Let $0<\kappa<\frac{1}{n+2}$ and define

$$
\Omega:=\left\{\xi \in 2 \pi R^{1-\kappa} \mathbb{Z}^{n}:|\xi| \leq R\right\}+B(0, \rho),
$$

where $\rho$, to be chosen later, will be sufficiently small. Letting $\theta \in \mathbb{S}^{n-1}$, we consider initial data $f_{\theta}$ defined by

$$
f_{\theta}(x)=e^{i \pi R \theta \cdot x} f(x)
$$

with $R$ large, where

$$
\widehat{f}=\frac{1}{\sqrt{|\Omega|}} \chi_{\Omega} .
$$

Note that $\left|\operatorname{supp} \widehat{f}_{\theta}\right|=|\Omega| \simeq R^{n \kappa}$, and $\left\|f_{\theta}\right\|_{2}=1$. In [3], it was shown that

$$
\left|e^{i \frac{t}{2 \pi R} \Delta} f(x)\right| \gtrsim \sqrt{|\Omega|} \text { for all }(x, t) \in \Lambda,
$$

where, taking $\varepsilon$ sufficiently small, $\Lambda$ is defined by

$$
\Lambda=\left\{x \in R^{\kappa-1} \mathbb{Z}^{n}:|x| \leq 2\right\}+B\left(0, \varepsilon R^{-1}\right) \times\left\{t \in R^{2 \kappa-1} \mathbb{Z}: 0<t<1\right\} .
$$

In fact they proved that the time can be taken in small intervals, however this will suffice for our needs. We provide the short proof of this for completeness. The idea is that the phase in the integrand in (2.1) never strays too far from zero modulo $2 \pi i$, and so the different pieces of the integral, corresponding to different pieces of $\Omega$, cannot cancel each other out.

We start by showing that

$$
x \cdot \xi \in 2 \pi \mathbb{Z}+\left(-\frac{1}{10}, \frac{1}{10}\right),
$$

provided that $\xi \in \Omega$ and $x \in R^{\kappa-1} \mathbb{Z}^{n} \cap B(0,2)+B\left(0, \varepsilon R^{-1}\right)$. To see this, we write

$$
\xi=2 \pi R^{1-\kappa} \ell+v, \quad \text { where } \ell \in \mathbb{Z}^{n}, \quad|\ell| \leq R^{\kappa}, \quad|v| \leq \rho
$$

and

$$
x=R^{\kappa-1} m+u, \quad \text { where } \quad m \in \mathbb{Z}^{n}, \quad|m| \leq 2 R^{1-\kappa}, \quad|u| \leq \varepsilon R^{-1},
$$

so that

$$
\begin{aligned}
x \cdot \xi & =\left(R^{\kappa-1} m+u\right) \cdot\left(2 \pi R^{1-\kappa} \ell+v\right) \\
& =2 \pi m \cdot \ell+R^{\kappa-1} m \cdot v+2 \pi R^{1-\kappa} \ell \cdot u+u \cdot v \\
& =: I_{1}+I_{2}+I_{3}+I_{4} .
\end{aligned}
$$

Since $I_{1} \in 2 \pi \mathbb{Z}^{n}$ and

$$
\left|I_{2}\right| \leq R^{1-\kappa} 2 R^{\kappa-1} \rho=2 \rho, \quad\left|I_{3}\right| \leq 2 \pi R^{1-\kappa} R^{\kappa} \frac{\varepsilon}{R}=2 \pi \varepsilon, \quad\left|I_{4}\right| \leq \rho \frac{\varepsilon}{R},
$$

we see that (2.17) holds by taking $\rho$ and $\varepsilon$ sufficiently small. On the other hand, we also have that

$$
\frac{t}{2 \pi R}|\xi|^{2} \in 2 \pi \mathbb{Z}+\left(-\frac{1}{10}, \frac{1}{10}\right)
$$

provided that $t \in R^{2 \kappa-1} \mathbb{Z} \cap(0,1)$. To see this, we write

$$
t=R^{2 \kappa-1} k, \quad \text { where } \quad k \in \mathbb{N}, \quad|k| \leq R^{1-2 \kappa},
$$


so that

$$
\begin{aligned}
\frac{t}{2 \pi R}|\xi|^{2} & =\frac{1}{2 \pi} R^{2(\kappa-1)} k\left|2 \pi R^{1-\kappa} \ell+v\right|^{2} \\
& =\frac{1}{2 \pi} R^{2(\kappa-1)} k\left(4 \pi^{2} R^{2(1-\kappa)}|\ell|^{2}+|v|^{2}+4 \pi R^{1-\kappa} \ell \cdot v\right) \\
& =: I I_{1}+I I_{2}+I I_{3},
\end{aligned}
$$

where $I I_{1} \in 2 \pi \mathbb{Z}$ while

and

$$
\left|I I_{2}\right| \leq \frac{1}{2 \pi} R^{2(\kappa-1)}|k||v|^{2} \leq \frac{1}{2 \pi} R^{2(\kappa-1)} R^{1-2 \kappa} \rho^{2}=\frac{\rho^{2}}{2 \pi R},
$$

$$
\left|I I_{3}\right| \leq 2 R^{2(\kappa-1)}|k| R^{1-\kappa}|\ell \cdot v| \leq 2 R^{\kappa-1}|k||\ell||v| \leq 2 R^{\kappa-1} R^{1-2 \kappa} R^{\kappa} \rho \leq 2 \rho,
$$

so that (2.19) is satisfied for sufficiently small $\rho$. Indeed altogether $|\rho|,|\varepsilon| \leq \frac{1}{100}$ is sufficient for our purposes. Now (2.17) and (2.19) imply that the phase in

$$
e^{i \frac{t}{2 \pi R} \Delta} f(x)=\frac{1}{(2 \pi)^{n / 2}} \frac{1}{\sqrt{|\Omega|}} \int_{\Omega} e^{i x \cdot \xi-i \frac{t}{2 \pi R}|\xi|^{2}} d \xi
$$

is close enough to zero modulo $2 \pi i$ as long as $(x, t) \in \Lambda$, yielding (2.16).

We now consider $\Gamma_{\theta, t} \subset \mathbb{R}^{n}$ defined by

$$
\Gamma_{\theta, t}:=\left\{x \in R^{\kappa-1} \mathbb{Z}^{n}:|x| \leq 2\right\}+B\left(t \theta, \varepsilon R^{-1}\right) .
$$

Noting that

$$
x \in \Gamma_{\theta, t} \quad \text { and } \quad t \in R^{2 \kappa-1} \mathbb{Z} \cap(0,1) \quad \Rightarrow \quad(x-t \theta, t) \in \Lambda,
$$

by (2.16) we have that

$$
\sup _{0<t<1}\left|e^{i \frac{t}{2 \pi R} \Delta} f(x-t \theta)\right| \gtrsim \sqrt{|\Omega|} \text { for all } x \in \Gamma_{\theta}:=\bigcup_{t \in R^{2 \kappa-1} \mathbb{Z} \cap(0,1)} \Gamma_{\theta, t} .
$$

By Galilean invariance, or direct calculation using the formula (2.1), we have

$$
\sup _{0<t<1}\left|e^{i \frac{t}{2 \pi R} \Delta} f_{\theta}(x)\right|=\sup _{0<t<1}\left|e^{i \frac{t}{2 \pi R} \Delta} f(x-t \theta)\right|,
$$

and we recall that $\left\|f_{\theta}\right\|_{2}=\|f\|_{2}=1$. Thus, by taking $f_{\theta}$ in (2.14), we obtain

$$
R^{s} \gtrsim \sqrt{|\Omega|\left|\Gamma_{\theta}\right|} \text {. }
$$

Since $\Gamma_{\theta}$ is nothing more that the $\varepsilon R^{-1}$-neighbourhood of $E_{\theta}$ from the second section, we can use Lemma 2.2 to take $\theta \in \mathbb{S}^{n-1}$ so that $\left|\Gamma_{\theta}\right| \geq|B(0,1 / 2)|$ for $R$ sufficiently large. As $|\Omega| \gtrsim R^{n \kappa}$, we obtain

$$
s \geq \frac{n \kappa}{2}
$$

and the proof is completed by letting $\kappa$ tend to $\frac{1}{n+2}$ as we may.

\section{Proof of Theorem 1.4}

The philosophy of this section will be somewhat similar to the previous one, however we will be less explicit this time and we will have no need for the ergodicity arguments. In fact, the argument follows more closely the philosophy of Iosevich and Rudnev [32] who counted lattice points on general surfaces. Counting such points is of course easier when the surface is the graph of a polynomial as in the previous section. For the sphere we take advantage of a number theoretic result that counts the number of ways the square of a large integer can be represented as a sum of squares.

First we will require the following simple lemma. 
Lemma 3.1. Let $0<\alpha \leq d$ and $0<\varepsilon, \kappa<1$. For all $R>1$, define

$$
\Lambda:=\left(R^{\kappa-1} \mathbb{Z}^{d}+B\left(0, \varepsilon R^{-1}\right)\right) \cap B(0,1)
$$

and $d \mu:=\chi_{\Lambda} d x$, where $d x$ is the Lebesgue measure on $\mathbb{R}^{d}$. Then

$$
c_{\alpha}(\mu) \lesssim \max \left(R^{-d \kappa}, R^{\alpha-d}\right) .
$$

Proof. Notice that $\Lambda$ is the union of approximately $R^{d(1-\kappa)}$ balls of radius $\varepsilon R^{-1}$ whose centres are pairwise separated by $R^{\kappa-1}$. We consider different cases depending on the size of $r$.

When $0<r \leq \varepsilon R^{-1}$, the ball $B(x, r)$ overlaps with only one ball $B_{j}$ of $\Lambda$. Thus

$$
\begin{aligned}
r^{-\alpha} \mu(B(x, r)) & \leq r^{-\alpha}\left|B(x, r) \cap B_{j}\right| \\
& \lesssim r^{-\alpha} \min \left(r^{d}, R^{-d}\right) \\
& \leq r^{d-\alpha} \lesssim R^{\alpha-d}
\end{aligned}
$$

On the other hand, if $\varepsilon R^{-1}<r \leq R^{\kappa-1}$, then $B(x, r)$ overlaps with at most $N \lesssim 2^{d}$ balls $B_{j}$, with $j=1, \ldots, N$, contained in $\Lambda$. Thus

$$
\begin{aligned}
r^{-\alpha} \mu(B(x, r)) & \leq r^{-\alpha}\left|B(x, r) \bigcap \bigcup_{j=1, \ldots, N} B_{j}\right| \\
& \lesssim r^{-\alpha}\left|B_{N}\right| \lesssim r^{-\alpha} R^{-d} \lesssim R^{\alpha-d}
\end{aligned}
$$

Finally, if $R^{\kappa-1}<r$, then $B(x, r)$ overlaps with at most $N \lesssim \min \left(r^{d}, 1\right) R^{d(1-\kappa)}$ balls $B_{j}$, with $j=1, \ldots, N$, contained in $\Lambda$. In this case,

$$
\begin{aligned}
r^{-\alpha} \mu(B(x, r)) & \leq r^{-\alpha}\left|B(x, r) \bigcap \bigcup_{j=1, \ldots, N} B_{j}\right| \\
& \lesssim r^{-\alpha} N R^{-d} \lesssim r^{-\alpha} \min \left(r^{d}, 1\right) R^{-d \kappa} .
\end{aligned}
$$

Now as

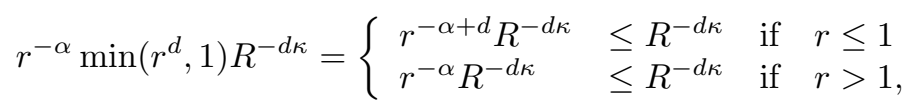

by collecting the three cases, the proof is complete.

Let $\sigma$ denote the surface measure on $\mathbb{S}^{d-1}$, and write $g=g_{1}-g_{2}+i\left(g_{3}-g_{4}\right)$, where each component $g_{j}$ is positive. Then by considering the positive measures $g_{j} \mu$, an application of the triangle inequality combined with (1.2) tells us that

$$
\|\widehat{g \mu}(R \cdot)\|_{L^{2}\left(\mathbb{S}^{d-1}\right)}^{2} \lesssim c_{\alpha}(\mu)\|\mu\| R^{-\beta}\|g\|_{L^{\infty}\left(\mathbb{S}^{d-1}\right)} .
$$

Thus, by duality, we are looking for an upper bound for the $\beta$ such that

$$
\left\|(f d \sigma)^{\vee}(R \cdot)\right\|_{L^{1}(d \mu)} \lesssim R^{-\beta / 2} \sqrt{c_{\alpha}(\mu)\|\mu\|}\|f\|_{L^{2}\left(\mathbb{S}^{d-1}\right)},
$$

where

$$
(f d \sigma)^{\vee}(x)=\frac{1}{(2 \pi)^{d / 2}} \int_{\mathbb{S}^{d-1}} e^{i \omega \cdot x} f(\omega) d \sigma(\omega) .
$$

We test this on the characteristic function associated to $\Omega$ defined by

$$
\Omega=\left\{\omega \in \mathbb{S}^{d-1}: \operatorname{dist}(\omega, \Gamma) \leq \rho R^{-1}\right\},
$$

where $\rho>0$ is sufficiently small, to be chosen later, and $\Gamma$ is defined by

$$
\Gamma=\left\{\omega \in \mathbb{S}^{d-1}: R^{\kappa} \omega \in 2 \pi \mathbb{Z}^{d}\right\},
$$

with $0<\kappa<1$. Considering $f$ defined by

$$
f=\frac{\chi_{\Omega}}{\sqrt{\sigma(\Omega)}}
$$

we have that $\|f\|_{L^{2}\left(\mathbb{S}^{d-1}\right)}=1$. 
Now it is well-known (see for example [24]) that for $d \geq 4$, there is a lower bound

$$
\# \Gamma \gtrsim R^{\kappa(d-2)},
$$

as long as $R$ is large enough and satisfies $\left(\frac{1}{2 \pi} R^{\kappa}\right)^{2} \in \mathbb{N}$. Thus, for these values of $R$, we have

$$
\sigma(\Omega) \gtrsim R^{\kappa(d-2)-(d-1)}
$$

We claim that

$$
\left|(f d \sigma)^{\vee}(R x)\right|=\left|\int_{\mathbb{S}^{d-1}} e^{i \omega \cdot R x} f(\omega) d \sigma(\omega)\right| \gtrsim \sqrt{\sigma(\Omega)} \quad \forall x \in \Lambda,
$$

where, taking $\varepsilon$ sufficiently small, $\Lambda$ is defined by

$$
\Lambda=\left(R^{\kappa-1} \mathbb{Z}^{d}+B\left(0, \varepsilon R^{-1}\right)\right) \cap B(0,1) .
$$

Again, the idea is that the phase of the integrand in (3.4) never strays too far from zero modulo $2 \pi i$, and so the different pieces of the integral, corresponding to different pieces of $\Omega$, do not cancel each other out.

More precisely we prove that

$$
\omega \cdot R x \in 2 \pi \mathbb{Z}+\left(-\frac{1}{10}, \frac{1}{10}\right),
$$

provided that $\omega \in \Omega$ and $x \in \Lambda$. To see this, we write

$$
\omega=2 \pi R^{-\kappa} \ell+v, \quad \text { where } \quad \ell \in \mathbb{Z}^{d}, \quad|\ell|=\frac{1}{2 \pi} R^{\kappa}, \quad|v|<\rho R^{-1}
$$

and

$$
x=R^{\kappa-1} m+u, \quad \text { where } \quad m \in \mathbb{Z}^{d}, \quad|m|<R^{1-\kappa}, \quad|u|<\varepsilon R^{-1},
$$

so that

$$
\begin{aligned}
\omega \cdot R x & =\left(2 \pi R^{-\kappa} \ell+v\right) \cdot\left(R^{\kappa} m+R u\right) \\
& =2 \pi \ell \cdot m+v \cdot R^{\kappa} m+2 \pi R^{1-\kappa} \ell \cdot u+v \cdot R u \\
& =: I_{1}+I_{2}+I_{3}+I_{4} .
\end{aligned}
$$

Since $I_{1} \in 2 \pi \mathbb{Z}$ and $\left|I_{2}\right| \leq \rho R^{-1} R^{\kappa} R^{1-\kappa}=\rho$,

$$
\left|I_{3}\right|<R^{1-\kappa} R^{\kappa} \varepsilon R^{-1}=\varepsilon \quad \text { and } \quad\left|I_{4}\right|<\rho R^{-1} R \varepsilon R^{-1}=\rho \varepsilon R^{-1},
$$

we see that (3.5) holds by taking $\rho$ and $\varepsilon$ sufficiently small. This implies that the phase in (3.4) is close enough to zero modulo $2 \pi i$ as long as $x \in \Lambda$, yielding the bound.

Now, defining $\mu$ by $d \mu=\chi_{\Lambda} d x$, where $d x$ is the Lebesgue measure in $\mathbb{R}^{d}$, and taking $\kappa=\frac{d-\alpha}{d}$, by Lemma 3.1 , we have

$$
c_{\alpha}(\mu) \lesssim R^{\alpha-d}=R^{-d \kappa}
$$

On the other hand,

$$
\|\mu\|=|\Lambda| \simeq R^{-d} R^{d(1-\kappa)}=R^{-d \kappa} .
$$

Now (3.2) combined with (3.4) tell us that

$$
\sqrt{\sigma(\Omega)}\|\mu\| \lesssim R^{-\beta / 2} \sqrt{c_{\alpha}(\mu)\|\mu\|},
$$

so that by plugging in (3.3), (3.6) and (3.7), we obtain

$$
R^{\frac{1}{2}(\kappa(d-2)-(d-1))} \lesssim R^{-\beta / 2} .
$$

Letting $R$ tend to infinity, we see that

$$
\beta \leq d-1-\kappa(d-2)=\alpha-1+\frac{2(d-\alpha)}{d},
$$

and so taking $\beta$ sufficiently close to $\beta_{d}(\alpha)$, the proof is complete. 


\section{Multilinear extension estimates}

Here we present the multilinear extension estimates due to Bennett, Carbery and Tao [5]. As we saw in the previous section, the extension operator, defined below, is also the adjoint of the operator that restricts the Fourier transform to a surface, and so these dual estimates are also referred to as restriction estimates. We consider the surfaces

$$
S:=\left\{(\xi, \phi(\xi)) \in \mathbb{R}^{d}:|\xi| \leq 1 / 2\right\}
$$

with $\phi(\xi)=-|\xi|^{2}$ or $\phi(\xi)=\sqrt{1-|\xi|^{2}}$. For a cap $\tau=\{(\xi, \phi(\xi)): \xi \in Q\} \subset S$ associated to a cube $Q$, we define the extension operator $T_{\tau}$ by

$$
T_{\tau} g(x, t)=\int_{Q} g(\xi) e^{i x \cdot \xi+i t \phi(\xi)} d \xi,
$$

Letting $Y(\xi) \in \mathbb{S}^{d-1}$ be the outward unit normal vector at a point $(\xi, \phi(\xi)) \in S$, we say that the caps $\tau_{1}, \ldots, \tau_{m}$ are $m$-transversal with constant $\theta>0$ if

$$
\left|Y\left(\xi_{1}\right) \wedge \cdots \wedge Y\left(\xi_{m}\right)\right|>\theta,
$$

for all $\xi_{1} \in Q_{1}, \ldots \xi_{m} \in Q_{m}$. In the following theorem, and throughout, $B_{R}$ will denote a ball of radius $R$ with arbitrary centre.

Theorem 4.1. [5] Let $d \geq 2, \varepsilon>0$ and let $\tau_{1}, \ldots, \tau_{d} \subset S$ be d-transversal caps with constant $\theta>0$. Then, for all $R>1$,

$$
\left\|\prod_{k=1}^{d} T_{\tau_{k}} g\right\|_{L^{\frac{2}{d-1}}\left(B_{R}\right)}^{\frac{2}{d-1}} \lesssim \mathfrak{c}(\theta) R^{\varepsilon} \prod_{k=1}^{d}\|g\|_{L^{2}\left(Q_{k}\right)}^{\frac{2}{d-1}} .
$$

The exact dependence of $\mathfrak{c}$ on $\theta$ is an interesting open question. The following lower-dimensional and discretised version was proven in [11, pp. 1250]. This is the version we will require in the following section.

Proposition 4.2. [11] Let $0<\varepsilon<\frac{1}{4 d}$ and let $\tau_{1}, \ldots, \tau_{m} \subset \tau$ be $m$-transversal caps with constant $\theta$, where $2 \leq m \leq d-1$. Let $\mathbb{V}_{m}$ be an $m$-dimensional subspace of $\mathbb{R}^{d}$ and let $Q_{j_{k}} \subset Q_{k}$ be disjoint cubes of side length $1 / K$ such that $\operatorname{dist}\left(Y(\xi), \mathbb{V}_{m}\right) \leq$ $1 / K$ for some $\xi \in Q_{j_{k}}$. Then, for all $K>1$,

$$
f_{B_{K}} \prod_{k=1}^{m}\left|\sum_{j_{k}} T_{\tau_{j_{k}}} g\right|^{\frac{2}{m-1}} \lesssim \mathfrak{c}(\theta) K^{\varepsilon}\left(f_{B_{K}} \prod_{k=1}^{m}\left(\sum_{j_{k}}\left|T_{\tau_{j_{k}}} g\right|^{2}\right)^{\frac{1}{2 m}}\right)^{\frac{2 m}{m-1}} .
$$

Due to rescaling arguments we will require these estimates for a slightly more general class of phases $\phi$. As we are only interested in the modulus of the extension operator, we are free to add and subtract constants to the phase $\phi$ and so we work instead with $\phi(\xi)=\sqrt{1-|\xi|^{2}}-1$ in the spherical case so that it looks very similar to the parabolic case. Then, for $\xi_{0} \in\left\{\xi \in \mathbb{R}^{d-1}:|\xi| \leq 1 / 2-\delta / 2\right\}$ and $0<\delta<1$, we define the scaling map $S_{\xi_{0}, \delta}$ by

$$
S_{\xi_{0}, \delta} \phi(\xi)=\delta^{-2}\left(\phi\left(\xi_{0}+\delta \xi\right)-\delta \nabla \phi\left(\xi_{0}\right) \cdot \xi-\phi\left(\xi_{0}\right)\right) .
$$

Note that the paraboloid is unchanged by this operation, and the sphere is altered only very mildly. The estimates of this section hold uniformly for all the extension operators defined with a phase obtained by applying the scaling map a finite number of times to $\phi$.

Finally we present a globalised-in-space version of Theorem 4.1 that we will need in the final sections. It follows by a standard localisation argument. 
Proposition 4.3. Let $\varepsilon>0, p=\frac{2 d}{d-1}$ and let $\tau_{1}, \ldots, \tau_{d} \subset S$ be $d$-transversal caps with constant $\theta>0$. Let $\{\Omega\}$ be a partition of $\mathbb{R}^{d-1}$ into cubes of side length $R$. Then, for all $R>1$,

$$
\left\|\prod_{k=1}^{d}\left|T_{\tau_{k}} g\right|^{\frac{1}{d}}\right\|_{L^{p}\left(\mathbb{R}^{d-1} \times(-R, R)\right)}^{2} \leq \sum_{\Omega}\left\|\prod_{k=1}^{d}\left|T_{\tau_{k}} g\right|^{\frac{1}{d}}\right\|_{L^{p}(\Omega \times(-R, R))}^{2} \lesssim \mathfrak{c}(\theta) R^{\varepsilon}\|g\|_{2}^{2} .
$$

Proof. Noting that the first inequality is nothing more than the inclusion $\ell^{2} \subset \ell^{p}$, it remains to prove the second which we rewrite as

$$
\sum_{\Omega}\left\|\prod_{k=1}^{d} T_{\tau_{k}} g\right\|_{L^{\frac{2}{d-1}}(\Omega \times(-R, R))}^{2 / d} \lesssim \mathfrak{c}(\theta) R^{\varepsilon}\|g\|_{2}^{2} .
$$

For this we write $g_{\Omega}=\left(\left(g \chi_{Q_{k}}\right)^{\vee} \chi_{\Omega^{*}}\right)^{\wedge}$ and $g_{\Omega^{c}}=g \chi_{Q_{k}}-g_{\Omega}$, where $\chi_{\Omega^{*}}$ is a Schwartz function adapted to the cube $\Omega^{*}$, with same centre as $\Omega$, but with side length

$$
10 \sup _{|\xi| \leq 1 / 2}|1+\nabla \phi(\xi)| R .
$$

Now that we have taken the support restriction inside the definition of the functions, we will consider the operator $T$ defined by

$$
T g(x, t):=\int_{\mathbb{R}^{d-1}} \psi(\xi) g(\xi) e^{i x \cdot \xi+i t \phi(\xi)} d \xi
$$

where $\psi$ is a Schwartz function supported in the unit ball and equal to one on $|\xi| \leq 1 / 2$. By applications of the triangle inequality it would then suffice to bound the main term as

$$
\sum_{\Omega}\left\|\prod_{k=1}^{d} T g_{\Omega}\right\|_{L^{\frac{2}{d-1}}(\Omega \times(-R, R))}^{2 / d} \lesssim \mathfrak{c}(\theta) R^{\varepsilon}\|g\|_{2}^{2},
$$

and prove other mixed inequalities, like for example

$$
\sum_{\Omega}\left\|T g_{\Omega^{c}} \prod_{k=2}^{d} T g_{\Omega}\right\|_{L^{d-1}(\Omega \times(-R, R))}^{2 / d} \lesssim\|g\|_{2}^{2}
$$

The main term is bounded directly using Theorem 4.1 and the finite overlapping of the frequency supports. For the second estimate we first note that by Hölder's inequality, followed by Bernstein's inequality (or Young's inequality given the compact frequency support and the reproducing formula that it yields, see below) and Plancherel's identity in the $x$-variable, the left-hand side of (4.3) is bounded by

$$
\sum_{\Omega}\left(\|\| T g_{\Omega^{c}}\left\|_{L^{2}(\Omega)}\right\| g_{\Omega} e^{i t \phi(\cdot)}\left\|_{2}^{d-1}\right\|_{L^{\frac{2}{d-1}}(|t| \leq R)}\right)^{2 / d} .
$$

Then by Hölder's inequality in the time integral, we see that this is bounded by

$$
R^{\frac{d-2}{d}} \sum_{\Omega}\left(\left\|T g_{\Omega^{c}}\right\|_{L^{2}(\Omega \times(-R, R))}\left\|g_{\Omega}\right\|_{2}^{d-1}\right)^{2 / d}
$$

A final application of Hölder's inequality in the sum, and the finite overlapping of the frequency supports, shows that this is bounded by

$$
R^{\frac{d-2}{d}}\|g\|_{2}\left(\sum_{\Omega}\left\|T g_{\Omega^{c}}\right\|_{L^{2}(\Omega \times(-R, R))}^{2}\right)^{1 / 2} .
$$

Thus in order to complete the proof of (4.3), we need only prove that

$$
\sum_{\Omega}\left\|T g_{\Omega^{c}}\right\|_{L^{2}(\Omega \times(-R, R))}^{2} \lesssim R^{-N}\|g\|_{2}^{2}
$$


for large enough $N \in \mathbb{N}$.

For this we write the operator as a convolution,

$$
T g_{\Omega^{c}}(x, t)=\int_{\mathbb{R}^{d-1}} \psi(\xi) g_{\Omega^{c}}(\xi) e^{i x \cdot \xi+i t \phi(\xi)} d \xi=\int_{z-x \notin \Omega^{*}} T[1](z, t)\left(g \chi_{Q_{1}}\right)^{\vee}(z-x) d z .
$$

Recalling the definitions (4.1) and (4.2), we have that $|z| \geq 2|\nabla \phi(\xi) t|$ when $(x, t) \in$ $\Omega \times(-R, R)$, so by repeated integration by parts we see that

$$
|T[1](z, t)| \lesssim C_{N}(1+|z|)^{-N-d-1},
$$

so that, for $(x, t) \in \Omega \times(-R, R)$, we have

$$
\left|T g_{\Omega^{c}}(x, t)\right| \lesssim R^{-N-1} \int_{\mathbb{R}^{d-1}}(1+|z|)^{-d}\left|\left(g \chi_{Q_{1}}\right)^{\vee}(z-x)\right| d z .
$$

Plugging this into (4.4), and integrating in time, we see that

$$
\begin{aligned}
\sum_{\Omega}\left\|T g_{\Omega^{c}}\right\|_{L^{2}(\Omega \times(-R, R))}^{2} & \lesssim R^{-N} \int_{\mathbb{R}^{d-1}}\left|\int(1+|z|)^{-d}\right|\left(g \chi_{Q_{1}}\right)^{\vee}(z-x)|d z|^{2} d x \\
& \lesssim R^{-N}\|g\|_{2}^{2},
\end{aligned}
$$

where the final inequality is by Young's inequality and the Plancherel identity. This completes the proof of (4.4) and thus (4.3), and the other mixed terms are bounded in an analogous manner.

\section{The Bourgain-Guth Decomposition}

In order to take advantage of the multilinear estimates, we must first decompose the operator in such a way that transversality presents itself. In order to take advantage of bilinear estimates, this can be done by employing something like a Whitney decomposition. A triumph of the work of Bourgain and Guth [11] was to achieve something similar in the multilinear setting. In fact they used the lower dimensional multilinear estimates of Proposition 4.2 in order to create the 'decomposition' (really it is an inequality) and in the coming sections we will need pointwise control of this. Indeed we will make essential use of the fact that the right-hand side of the inequality is almost constant at certain scales. As they point out, this only holds after mollifications, and the final decomposition is obtained by an iteration. In this section, we keep track of some of the details that they omitted so as to check that these approximations, as well as the lack of control of the constant $\mathfrak{c}$ from the previous section, do not feedback in an uncontrolled way.

Let $Q \subset\left\{\xi \in \mathbb{R}^{d-1}:|\xi| \leq 1 / 2\right\}$ be a box of side length $\delta$ and let $\tau$ denote the associated cap. Take $0<\varepsilon<\frac{1}{4 d}$ and $R>1$ and introduce $d$ different scales

$$
R^{1 / \mathfrak{c}(\varepsilon)}<K_{2}<\cdots<K_{d+1}<R^{\varepsilon}
$$

that satisfy $K_{m}^{8 m} \mathfrak{c}\left(K_{m}^{-m}\right) \leq K_{m+1}^{\varepsilon}$, where $\mathfrak{c}(\varepsilon) \geq 1 / \varepsilon^{2 d}$ dominates the constant from the previous section. As long as it does not blow up at zero in a very unexpectedly fast way, it would suffice to take $K_{m} \simeq R^{\varepsilon^{2(d+2-m)}}$. One can calculate that we also have $R^{\frac{1}{\varepsilon(\varepsilon)}} K_{m} \leq K_{m+1}$.

Take a partition $\left\{Q_{2, \ell}\right\}$ of $Q$ made of pairwise disjoint cubes of side length $\delta / K_{2}$ and centered in $\xi_{\ell}$. Then, for all $m=3, \ldots, d$, define recursively a sub-partition $\left\{Q_{m, j}\right\}$ made by pairwise disjoint cubes of side length $\delta / K_{m}$ and centered at $\xi_{j}$ in such a way that for every $Q_{m, j}$ there exists an $Q_{m-1, \ell}$ that contains it. For this we need to suppose that $R>2^{\mathfrak{c}(\varepsilon)}$ in order to have room to choose the scales appropriately, and so this is assumed from now on. 
We say that the caps $\tau_{m, j}$ associated to $Q_{m, j}$ are at scale $\delta / K_{m}$. Recalling that

$$
T_{\tau_{m, j}} g(x, t)=\int_{Q_{m, j}} g(\xi) e^{i x \cdot \xi+i t \phi(\xi)} d \xi,
$$

for each $m=2, \ldots, d$, we have

$$
T_{\tau} g=\sum_{j} T_{\tau_{m, j}} g
$$

We will also need a restricted version of $T_{\tau_{m, j}}$. Let $\mathbb{V}_{m}$ be an $m$-dimensional subspace of $\mathbb{R}^{d}$ and define

$$
T_{\tau_{m, j}}^{\mathbb{V}_{m}} g:=\sum_{\substack{v \subset \tau_{m, j} \\ v \in V_{m, \tau}}} T_{v} g
$$

where $V_{m, \tau}:=\left\{\tau_{m+1, \ell} \subset \tau: \operatorname{dist}\left(Y(\xi), \mathbb{V}_{m}\right) \leq \delta / K_{m+1}\right.$ for some $\left.\xi \in Q_{m+1, \ell}\right\}$.

The following pointwise estimate $[11$, pp. 1256] will be a key ingredient:

$$
\begin{aligned}
\left|T_{\tau} g(x, t)\right| \lesssim K_{d}^{2 d} \max _{\tau_{1}, \ldots, \tau_{d} \subset \tau} \prod_{k=1}^{d}\left|T_{\tau_{k}} g(x, t)\right|^{\frac{1}{d}} \\
\quad+\sum_{m=2}^{d-1} K_{m}^{2 m} \max _{\substack{\mathbb{V}_{m} \\
\tau_{1}, \ldots, \tau_{m} \subset \tau}} \prod_{k=1}^{m}\left|T_{\tau_{k}}^{\mathbb{V}_{m}} g(x, t)\right|^{\frac{1}{m}}+\sum_{m=2}^{d} \max _{\tau_{m} \subset \tau}\left|T_{\tau_{m}} g(x, t)\right| .
\end{aligned}
$$

Here the caps $\tau_{1}, \ldots, \tau_{m}$ in the first two maxima are $m$-transversal at scale $\delta / K_{m}$, and the final maximum is over caps $\tau_{m}$ at scale $\delta / K_{m}$. This is proved by iterating the following dichotomy: either the operator is bounded by a product of $m+1$ operators associated to transversal caps, or it is not, in which case, given $m$ caps where the operator is large and the hyperplane $\mathbb{V}_{m}$ that their normals generate, the operators associated to the caps with normal lying outside of $\mathbb{V}_{m}$ must be small.

The uncertainty principle tells us that the terms should be essentially constant at different scales $\delta / K$. This can be formalised by replacing them with suitable majorant functions. Indeed, define the dual set $\tau^{\prime}$ to be the $d$-dimensional cuboid with dimensions $\delta^{-1} \times \ldots \times \delta^{-1} \times \delta^{-2}$ centred at the origin, and with long side normal to $\tau$ (pointing in the direction of the normal $Y_{\tau}$ to the centre of a cap $\tau$ ). The scaled version $K \tau^{\prime}$ denotes the similar set but with dimensions $K \delta^{-1} \times \ldots \times K \delta^{-1} \times K \delta^{-2}$. Let $\widehat{\psi}=\widehat{\psi}_{\mathrm{o}} * \widehat{\psi}_{\mathrm{o}}$ be a smooth radially symmetric cut-off function, supported on $B(0, d) \subset \mathbb{R}^{d}$ and equal to one on $B(0, \sqrt{d}) \subset \mathbb{R}^{d}$ and let $\psi_{K \tau^{\prime}}$ denote the scaled version of $\psi$ adapted to $K \tau^{\prime}$. By this we mean that

$$
\psi_{K \tau^{\prime}}(x, t):=\frac{\delta^{d+1}}{K^{d}} \psi\left(\frac{\delta x^{\prime}}{K}, \frac{\delta^{2} t^{\prime}}{K}\right), \quad\left(x^{\prime}, t^{\prime}\right)=\Lambda_{\tau}(x, t),
$$

where $\Lambda_{\tau} \in S O(d)$ and $\Lambda_{\tau}\left(Y_{\tau}\right)=(0, \ldots, 0,1)$. By the modulated reproducing formula,

$$
\left|T_{\tau} g\right| \leq\left|T_{\tau} g\right| *\left|\psi_{\tau^{\prime}}\right|
$$

and one can also calculate (see Lemma 8.6 of the appendix) that

$$
\left|T_{\tau} g\right| \lesssim\left(\left|T_{\tau} g\right|^{\frac{1}{m}} *\left|\psi_{\tau^{\prime}}\right|^{\frac{1}{m}}\right)^{m}
$$

for any $m \geq 1$. This yields

$$
\left|T_{\tau} g\right|^{\frac{1}{m}} \lesssim\left|T_{\tau} g\right|^{\frac{1}{m}} * \zeta_{\tau^{\prime}}, \quad \zeta(x, t):=\left(1+|x|^{2}+|t|^{2}\right)^{-\mathfrak{c}(\varepsilon)}
$$

and as $\zeta_{\tau^{\prime}}$ is essentially constant on translates of $\tau^{\prime}$, which is a property that is preserved under convolution, we have majorised by an essentially constant function. 
By elementary trigonometry one sees that $\frac{1}{2} K_{m} \tau^{\prime} \subset v^{\prime}$ whenever $v \subset \tau$ is at scale $\delta / K_{m}$, so that the dual of the latter is contained in the former and so

$$
\left|T_{v}^{\mathbb{V}_{m}} g\right|^{\frac{1}{m}} \lesssim\left|T_{v}^{\mathbb{V}_{m}} g\right|^{\frac{1}{m}} * \zeta_{K_{m} \tau^{\prime}}
$$

Using these observations, (5.1) can be rewritten as

$$
\begin{aligned}
\left|T_{\tau} g\right| & \lesssim K_{d}^{2 d} \max _{\tau_{1}, \ldots, \tau_{d}} \prod_{k=1}^{d}\left|T_{\tau_{k}} g\right|^{\frac{1}{d}} * \zeta_{\tau_{k}^{\prime}} \\
& +\sum_{m=2}^{d-1} K_{m}^{2 m} \max _{\mathbb{V}_{\mathbb{V}_{m}}, \ldots, \tau_{m}} \prod_{k=1}^{m}\left|T_{\tau_{k}}^{\mathbb{V}_{m}} g\right|^{\frac{1}{m}} * \zeta_{K_{m} \tau^{\prime}}+\sum_{m=1}^{d-1} \max _{\tau_{m+1}}\left|T_{\tau_{m+1}} g\right| * \zeta_{\tau_{m+1}^{\prime}}
\end{aligned}
$$

where as before $\tau_{1}, \ldots, \tau_{m} \subset \tau$ are $m$-transversal caps at scale $\delta / K_{m}$ and the maximum in the last term is taken over caps of size $\delta / K_{m+1}$.

Remark 5.1. The maximum over $\tau_{1}, \ldots, \tau_{m}, \mathbb{V}_{m}$ depends on the value of $(x, t)$, but we can now choose the same $\tau_{1}, \ldots, \tau_{m}, \mathbb{V}_{m}$ for all $(x, t)$ in a translate of $K_{m} \tau^{\prime}$. In fact, given the dichotomy with which the initial decomposition is obtained, $\mathbb{V}_{m}$ can be chosen to be the same in any translate of $K_{m+1} \tau^{\prime}$. This is because we only need to consider this lower dimensional case in the absence of $m+1$ transversal caps for which the operator is large. These caps are at scale $K_{m+1}$ and so the definition of the subspace $\mathbb{V}_{m}$ can be taken uniformly at that scale.

Definition 5.1. Set $\Phi_{\tau, \mathbb{V}_{1}, \tau_{2}}=1$ and, for $m=2, \ldots, d-1$, define

$$
\Phi_{\tau, \mathbb{V}_{m}, \tau_{m+1}}:=\frac{K_{m}^{2 m} \max _{\tau_{1}, \ldots, \tau_{m} \subset \tau} \prod_{k=1}^{m}\left|T_{\tau_{k}}^{\mathbb{V}_{m}} g\right|^{\frac{1}{m}} * \zeta_{K_{m} \tau^{\prime}}+\left|T_{\tau_{m+1}} g\right| * \zeta_{\tau_{m+1}^{\prime}}}{\left(\sum_{v \in V_{m, \tau} \cup\left\{\tau_{m+1}\right\}}\left(\left|T_{v} g\right| * \zeta_{v^{\prime}}\right)^{2}\right)^{1 / 2}+R^{-1 / \varepsilon}\|g\|_{L^{2}}} .
$$

With this function, the decomposition (5.3) can be rewritten as

$$
\begin{aligned}
\left|T_{\tau} g\right| \lesssim & K_{d}^{2 d} \max _{\tau_{1}, \ldots, \tau_{d} \subset \tau} \prod_{k=1}^{d}\left|T_{\tau_{k}} g\right|^{\frac{1}{d}} * \zeta_{\tau_{k}^{\prime}} \\
& +\sum_{m=1}^{d-1} \max _{\mathbb{V}_{m}, \tau_{m+1}} \Phi_{\tau, \mathbb{V}_{m}, \tau_{m+1}}\left(\sum_{v \in V_{m, \tau} \cup\left\{\tau_{m+1}\right\}}\left(\left|T_{v} g\right| * \zeta_{v^{\prime}}\right)^{2}\right)^{1 / 2}+\mathcal{R}_{\tau}(g),
\end{aligned}
$$

where the remainder term $\mathcal{R}_{\tau}(g)$ is defined by

$$
\mathcal{R}_{\tau}(g):=R^{-1 / \varepsilon} \sum_{m=1}^{d-1} \max _{\mathbb{V}_{m}, \tau_{m+1}} \Phi_{\tau, \mathbb{V}_{m}, \tau_{m+1}}\|g\|_{L^{2}} .
$$

Although $\Phi_{\tau, \mathbb{V}_{m}, \tau_{m+1}}$ looks complicated, we will no longer care about its explicit form, and focus instead on its properties. These properties, one of which we prove now using the multilinear extension estimate, hold uniformly for all hyperplanes $\mathbb{V}_{m}$ and caps $\tau_{m+1}$ at scale $\delta / K_{m+1}$.

Lemma 5.2. Let $0<\varepsilon<\frac{1}{4 d}$ and $0<\delta \leq 1$. Let $\tau_{1}, \ldots, \tau_{m}$ be $m$-transversal caps at scale $\delta / K_{m}$ and let $\tau$ be a cap at scale $\delta$ that contains them. Then, for all $\mathbb{V}_{m} \subset \mathbb{R}^{d}$ and $a \in \mathbb{R}^{d}$,

$$
\begin{gathered}
f_{a+K_{m+1} \tau^{\prime}}\left(\prod_{k=1}^{m}\left|T_{\tau_{k}}^{\mathbb{V}_{m}} g\right|^{\frac{1}{m}} * \zeta_{K_{m} \tau^{\prime}}\right)^{\frac{2 m}{m-1}} \\
\lesssim \mathfrak{c}\left(K_{m}^{-m}\right) K_{m+1}^{\varepsilon}\left(\sum_{v \in V_{m, \tau}}\left(\left|T_{v} g\right| * \zeta_{v^{\prime}}\right)^{2}(a)\right)^{\frac{m}{m-1}}+\left(R^{-1 / \varepsilon}\|g\|_{L^{2}}\right)^{\frac{2 m}{m-1}} .
\end{gathered}
$$


Proof. Denoting $q:=\frac{2 m}{m-1}$, by the trivial bound $\left\|T_{\tau_{m+1}} g\right\|_{L^{\infty}} \leq K_{m+1}^{-n / 2}\|g\|_{L^{2}}$, and the definition of the $K_{m}$, this would follow from the slightly stronger estimate

$$
\begin{gathered}
f_{a+K_{m+1} \tau^{\prime}}\left(\prod_{k=1}^{m}\left|T_{\tau_{k}}^{\mathbb{V}_{m}} g\right|^{\frac{1}{m}} * \zeta_{K_{m} \tau^{\prime}}\right)^{q} \lesssim \\
\mathfrak{c}\left(K_{m}^{-m}\right) K_{m+1}^{\varepsilon}\left(\sum_{v \in V_{m, \tau}}\left(\left|T_{v} g\right| * \zeta_{v^{\prime}}\right)^{2}(a)\right)^{q / 2}+\left(\left(\frac{K_{m}}{K_{m+1}}\right)^{\mathfrak{c}(\varepsilon)} K_{m+1}^{n / 2} \max _{v \in V_{m, \tau}}\left\|T_{v} g\right\|_{\infty}\right)^{q} .
\end{gathered}
$$

By scaling as in the proof of the forthcoming Lemma 6.3, it will be enough to prove this with $\delta=1$, so we can replace $a+K_{m+1} \tau^{\prime}$ by $B_{K_{m+1}}$ centred at $a$. By Hölder's inequality and Fubini's theorem, we see that

$$
\begin{aligned}
& f_{B_{K_{m}+1}}\left(\prod_{k=1}^{m} \int\left|T_{\tau_{k}}^{\mathbb{V}_{m}} g\left((x, t)-y_{k}\right)\right|^{\frac{1}{m}} \zeta_{K_{m} \tau^{\prime}}^{1 / q}\left(y_{k}\right) \zeta_{K_{m} \tau^{\prime}}^{1-1 / q}\left(y_{k}\right) d y_{k}\right)^{q} d x d t \\
\lesssim & \int\left(f_{B_{K_{m}+1}} \prod_{k=1}^{m}\left|T_{\tau_{k}}^{\mathbb{V}_{m}} g\left((x, t)-y_{k}\right)\right|^{\frac{2}{m-1}} d x d t\right) w(y) d y,
\end{aligned}
$$

where $\prod_{k=1}^{m} \zeta_{K_{m} \tau^{\prime}}\left(y_{k}\right) d y_{1} \ldots d y_{m}=: w(y) d y$. Then by Proposition 4.2 (with $K=$ $K_{m+1}$ and $\theta=K_{m}^{-m}$ ), Hölder's inequality and Fubini, this is bounded by a constant multiple of

$$
\begin{aligned}
& \mathfrak{c}\left(K_{m}^{-m}\right) K_{m+1}^{\varepsilon} \int\left(f_{B_{K_{m}+1}} \prod_{k=1}^{m}\left(\sum_{\substack{v \in \tau_{k} \\
v \in V_{m, \tau}}}\left|T_{v} g\left((x, t)-y_{k}\right)\right|^{2}\right)^{\frac{1}{2 m}} d x d t\right)^{q} w(y) d y \\
& \lesssim \mathfrak{c}\left(K_{m}^{-m}\right) K_{m+1}^{\varepsilon} f_{B_{K_{m+1}}} \int \prod_{k=1}^{m}\left(\sum_{\substack{v \subset \tau_{k} \\
v \in V_{m, \tau}}}\left|T_{v} g\left((x, t)-y_{k}\right)\right|^{2}\right)^{\frac{q}{2 m}} w(y) d y d x d t .
\end{aligned}
$$

By Hölder's inequality again and the reproducing formula, we can bound this as

$$
\begin{aligned}
& \lesssim \mathfrak{c}\left(K_{m}^{-m}\right) K_{m+1}^{\varepsilon} f_{B_{K_{m}+1}} \prod_{k=1}^{m}\left(\sum_{\substack{v \in \tau_{k} \\
v \in V_{m, \tau}}}\left(\left|T_{v} g\right| * \zeta_{v^{\prime}}\right)^{2} * \zeta_{K_{m} \tau^{\prime}}(x, t)\right)^{\frac{q}{2 m}} d x d t \\
& \leq \mathfrak{c}\left(K_{m}^{-m}\right) K_{m+1}^{\varepsilon} f_{B_{K_{m+1}}}\left(\sum_{v \in V_{m, \tau}}\left(\left|T_{v} g\right| * \zeta_{v^{\prime}}\right)^{2} * \zeta_{K_{m} \tau^{\prime}}(x, t)\right)^{q / 2} d x d t .
\end{aligned}
$$

Finally we can apply Lemma 8.7 of the appendix, with $K=K_{m+1}$ and $K^{\prime}=K_{m}$, to conclude that this is bounded by a constant multiple of

$$
\mathfrak{c}\left(K_{m}^{-m}\right) K_{m+1}^{\varepsilon}\left(\sum_{v \in V_{m}, \tau}\left(\left|T_{v} g\right| * \zeta_{v^{\prime}}\right)^{2}(a)\right)^{q / 2}+\left(\left(\frac{K_{m}}{K_{m+1}}\right)^{\mathfrak{c}(\varepsilon)} K_{m+1}^{n / 2} \max _{v}\left\|T_{v} g\right\|_{L^{\infty}}\right)^{q} .
$$

The chain of inequalities yields (5.5) and hence the result.

Property 5.1. It is clear that $\Phi_{\tau, \mathbb{V}_{m}, \tau_{m+1}}$ is essentially constant on translates of $K_{m} \tau^{\prime}$. Given that by definition $K_{m}^{\frac{4 m^{2}}{m-1}} \mathfrak{c}\left(K_{m}^{-m}\right) \leq K_{m+1}^{\varepsilon}$, Lemma 5.2 yields

$$
f_{a+K_{m+1} \tau^{\prime}} \Phi_{\tau, \mathbb{V}_{m}, \tau_{m+1}}^{\frac{2 m}{m-1}} \lesssim K_{m+1}^{2 \varepsilon}
$$

where $m=2, \ldots, d-1$. By Hölder's inequality this also implies that

$$
f_{a+K_{m+1} \tau^{\prime}} \Phi_{\tau, \mathbb{V}_{m}, \tau_{m+1}}^{\frac{2(d-1)}{d-2}} \lesssim K_{m+1}^{2 \varepsilon}
$$


uniformly over all $a \in \mathbb{R}^{d}, \mathbb{V}_{m} \subset \mathbb{R}^{d}$ and $\tau_{m+1} \subset \tau$ at scale $\delta / K_{m+1}$.

We could have convolved both sides of (5.3) with $\zeta_{\tau^{\prime}}$, before introducing the function $\Phi_{\tau, \mathbb{V}_{m}, \tau_{m+1}}$. In order to then replace the double convolutions on the right-hand side by single convolutions we again use Lemma 8.7 of the appendix. Introducing $\Phi_{\tau, \mathbb{V}_{m}, \tau_{m+1}}$ after this process, we can also write

$$
\begin{aligned}
\left|T_{\tau} g\right| * \zeta_{\tau^{\prime}} \lesssim & K_{d}^{2 d} \max _{\tau_{1}, \ldots, \tau_{d}} \prod_{k=1}^{d}\left|T_{\tau_{k}} g\right|^{\frac{1}{d}} * \zeta_{\tau_{k}^{\prime}} \\
& +\sum_{m=2}^{d-1} \max _{\mathbb{V}_{m}, \tau_{m+1}} \Phi_{\tau, \mathbb{V}_{m}, \tau_{m+1}}\left(\sum_{v \in V_{m, \tau} \cup\left\{\tau_{m+1}\right\}}\left(\left|T_{v} g\right| * \zeta_{v^{\prime}}\right)^{2}\right)^{1 / 2}+\mathcal{R}_{\tau} .
\end{aligned}
$$

As the terms on the right-hand side have the same form as the left-hand side at a different scale, we can iterate this inequality to obtain the following theorem. From now on we write $\tau \sim \delta / K$ if $\tau$ is a cap at scale $\delta / K$.

Definition 5.3. Define $\Psi_{v}$ recursively by

$$
\begin{array}{llrl}
\Psi_{v}:=1 & v \sim 1, & \\
\Psi_{v}:=\Psi_{\tau} \max _{\mathbb{V}_{m}, \tau_{m+1}} \Phi_{\tau, \mathbb{V}_{m}, \tau_{m+1}} & v \subset \tau, \quad v \sim \delta / K_{m+1}, \quad \tau \sim \delta .
\end{array}
$$

We keep track of the maximal number of caps in the following sets $E_{\delta}$ as this information is used when proving linear restriction estimates. However the cardinality will have no consequence in this article - it will only be important that the caps of these sets are disjoint.

Proposition 5.4. Let $0<\varepsilon<\frac{1}{4 d}$ and let $S=\{(\xi, \phi(\xi)):|\xi| \leq 1 / 2\}$. Then, for all $N \in \mathbb{N}$,

$$
\begin{aligned}
\left|T_{S} g\right| \lesssim_{N} & K_{d}^{2 d} \sum_{K_{2}^{-N}<\delta \leq 1} \max _{E_{\delta}}\left(\sum_{\tau \in E_{\delta}} \Psi_{\tau}^{2}\left(\max _{\tau_{1}, \ldots, \tau_{d} \subset \tau} \prod_{k=1}^{d}\left|T_{\tau_{k}} g\right|^{\frac{1}{d}} * \zeta_{\tau_{k}^{\prime}}\right)^{2}\right)^{1 / 2} \\
& +\sum_{K_{2}^{-N} K_{d}^{-1}<\delta \leq K_{2}^{-N}} \max _{E_{\delta}}\left(\sum_{\tau \in E_{\delta}} \Psi_{\tau}^{2}\left(\left|T_{\tau} g\right| * \zeta_{\tau^{\prime}}\right)^{2}\right)^{1 / 2} \\
& +\sum_{K_{2}^{-N} K_{d}^{-1}<\delta \leq 1} \max _{E_{\delta}}\left(\sum_{\tau \in E_{\delta}} \Psi_{\tau}^{2}\right)^{1 / 2} R^{-1 / \varepsilon}\|g\|_{L^{2}}
\end{aligned}
$$

provided supp $g \subset\left\{\xi \in \mathbb{R}^{d-1}:|\xi| \leq 1 / 2\right\}$. Here $\delta$ is restricted to taking values of the form $K_{2}^{-\gamma_{2}} \cdot \ldots \cdot K_{d}^{-\gamma_{d}}$ with $\gamma_{2}, \ldots, \gamma_{d} \in \mathbb{N} \cup\{0\}$ and $\tau_{1}, \ldots, \tau_{d}$ are $d$-transversal caps at scale $\delta / K_{d}$. The sets $E_{\delta}$ consist of at most $4^{N} \delta^{2-d}$ disjoint caps at scale $\delta$.

Proof. When $N=1$, there is only one term in the sum over $K_{2}^{-N}<\delta \leq 1$ and the inequality follows from (5.4) at scale one. So we proceed by induction on $N$.

Suppose the inequality is true for $N$. Note that if it were not for the upper bound on $\delta$ in the second sum on the right-hand side, the inequality with $N+1$ would immediately follow from the $N$ th version. Thus it remains to bound the part of the sum that appears in the $N$ th version that does not appear in the version with $N+1$;

$$
\sum_{K_{2}^{-(N+1)}<\delta \leq K_{2}^{-N}} \max _{E_{\delta}}\left(\sum_{\tau \in E_{\delta}} \Psi_{\tau}^{2}\left(\left|T_{\tau} g\right| * \zeta_{\tau^{\prime}}\right)^{2}\right)^{1 / 2} .
$$


Applying (5.6) to the summands, this is bounded by a constant multiple of

$$
\begin{aligned}
& \sum_{K_{2}^{-(N+1)}<\delta \leq K_{2}^{-N}} \max _{E_{\delta}}\left(\sum_{\tau \in E_{\delta}} \Psi_{\tau}^{2}\left(K_{d}^{2 d} \max _{\tau_{1}, \ldots, \tau_{d} \subset \tau} \prod_{k=1}^{d}\left|T_{\tau_{k}} g\right|^{\frac{1}{d}} * \zeta_{\tau_{k}^{\prime}}\right)^{2}\right)^{1 / 2}
\end{aligned}
$$

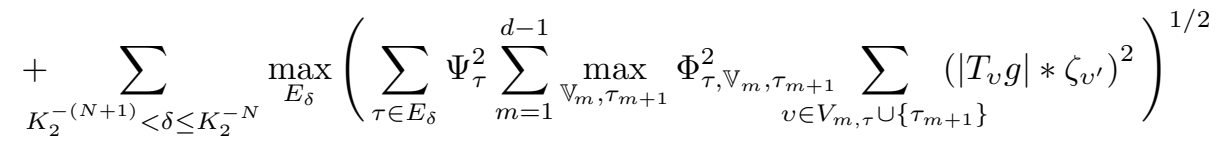

$$
\begin{aligned}
& +\sum_{K_{2}^{-(N+1)}<\delta \leq K_{2}^{-N}} \max _{E_{\delta}}\left(\sum_{\tau \in E_{\delta}} \Psi_{\tau}^{2} \mathcal{R}_{\tau}^{2}(g)\right)^{1 / 2}
\end{aligned}
$$

Here $\tau_{1}, \ldots, \tau_{d}$ are $d$-transversal caps of size $\delta / K_{d}$ and $V_{m, \tau}$ is the set of all the caps $v \subset \tau$ of size $\delta / K_{m+1}$ and such that $\operatorname{dist}\left(Y(\xi), \mathbb{V}_{m}\right) \leq \delta / K_{m+1}$ for some $\xi$ in the orthogonal projection of $v$. The first term is clearly acceptable and, by the definitions of $\Psi_{\tau}$ and $\mathcal{R}_{\tau}$, we can bound the other two as

$$
\begin{aligned}
& \sum_{K_{2}^{-(N+1)}<\delta \leq K_{2}^{-N}} \max _{E_{\delta}}\left(\sum_{\tau \in E_{\delta}} \sum_{m=2}^{d-1} \sum_{v \in V_{m, \tau} \cup\left\{\tau_{m+1}\right\}} \Psi_{v}^{2}\left(\left|T_{v} g\right| * \zeta_{v^{\prime}}\right)^{2}\right)^{1 / 2} \\
& +\sum_{K_{2}^{-(N+1)}<\delta \leq K_{2}^{-N}} n \max _{E_{\delta}}\left(\sum_{\tau \in E_{\delta}: v \subset \tau} \Psi_{v}^{2} R^{-2 / \varepsilon}\|g\|_{L^{2}}^{2}\right)^{1 / 2} \cdot
\end{aligned}
$$

Using the induction hypothesis again, there are at most

$$
4^{N} \delta^{2-d} 4\left(\delta /\left(\delta / K_{m}\right)\right)^{d-2}=4^{N+1}\left(\delta / K_{m}\right)^{2-d}
$$

terms in the product $E_{\delta} \times V_{m, \tau} \cup\left\{\tau_{m+1}\right\}$, so we shift the scale and bound this by a constant multiple of

$$
\begin{aligned}
& \sum_{K_{2}^{-(N+1)} K_{d}^{-1}<\delta \leq K_{2}^{-(N+1)}} \max _{E_{\delta}}\left(\sum_{v \in E_{\delta}} \Psi_{v}^{2}\left(\left|T_{v} g\right| * \zeta_{v^{\prime}}\right)^{2}\right)^{1 / 2} \\
& +\sum_{K_{2}^{-(N+1)}} \max _{K_{d}^{-1}<\delta \leq K_{2}^{-(N+1)}}\left(\sum_{E_{\delta}} \Psi_{v}^{2}\right)^{1 / 2} R^{-1 / \varepsilon}\|g\|_{L^{2}} .
\end{aligned}
$$

This is also acceptable and so the proof is complete.

Definition 5.5. If $\tau$ is a cap at scale $K_{2}^{-\gamma_{2}} \cdots K_{d}^{-\gamma_{d}}$ we write $l(\tau):=\sum_{j=2}^{d} \gamma_{j}$.

The functions $\Psi_{\tau}$ also have good essentially constant properties, that we record in the following proposition.

Proposition 5.6. Let $0<\varepsilon<\frac{1}{4 d}$. Then the functions $\Psi_{\tau}$ are essentially constant at scale one. Moreover, for all $a \in \mathbb{R}^{d}$,

$$
f_{a+\tau^{\prime}} \Psi_{\tau}^{\frac{2(d-1)}{d-2}}(x, t) d x d t \lesssim_{l(\tau)}\left|\tau^{\prime}\right|^{\varepsilon} .
$$

Proof. The essentially constant property is an immediate consequence of the definition and the corresponding property for $\Phi_{v}$ with $v \subset \tau$, so it remains to prove the averaged property.

If $\tau \sim 1$, then $\Psi_{\tau}:=1$ and the estimate is trivially satisfied. If $v \sim 1 / K_{m+1}$, then $\Psi_{v}:=\max _{\mathbb{V}_{m}, \tau_{m+1}} \Phi_{\tau, \mathbb{V}_{m}, \tau_{m+1}}$ where $v \subset \tau \sim 1$ and we can cover $a+v^{\prime}$ with 
a family of translates of $K_{m+1} \tau^{\prime}$ which are essentially balls $B_{j}$ of diameter $K_{m+1}$. We can of course do this in such a way that

$$
\bigcup_{j} B_{j} \subset a+4 v^{\prime}
$$

Then we have

$$
\begin{aligned}
\int_{a+v^{\prime}} \Psi_{v}^{q} & \leq \sum_{j} \int_{B_{j}} \max _{m}, \tau_{m+1} \\
& \lesssim \sum_{j}\left|B_{j}\right| f_{B_{j}} \max _{\mathbb{V}_{m}, \tau_{m+1}, \tau_{m+1}} \Phi_{\tau, \mathbb{V}_{m}, \tau_{m+1}}^{q}
\end{aligned}
$$

Recalling Remark 5.1, in fact we have the same $\mathbb{V}_{m}$ for all $(x, t) \in B_{j}$. Similarly as $\Phi_{\tau, \mathbb{V}_{m}, \tau_{m+1}}$ is essentially constant on $B_{j}$ we can suppose that the maximum is attained on the same $\tau_{m+1}$ for a given $B_{j}$. Thus, taking $q=\frac{2(d-1)}{d-2}$, by Property 5.1 we obtain

$$
\int_{a+v^{\prime}} \Psi_{v}^{q} \lesssim \sum_{B_{j}}\left|B_{j}\right| K_{m+1}^{2 \varepsilon} \lesssim\left|v^{\prime}\right| K_{m+1}^{2 \varepsilon} \leq\left|v^{\prime}\right|^{1+\varepsilon}
$$

as claimed.

We have proved the proposition for $\tau$ such that $l(\tau)=0$ or 1 . Thus we can proceed by induction on this quantity. Supposing that we have the estimate for $\tau$ such that $l(\tau)=N$, it will suffice to prove the estimate for $v$ such that $l(v)=N+1$. That is we suppose that

$$
f_{a+\tau^{\prime}} \Psi_{\tau}^{q} \lesssim\left|\tau^{\prime}\right|^{\varepsilon}, \quad a \in \mathbb{R}^{d}
$$

and attempt to prove the same for $v$ at scale $\delta / K_{m+1}$ such that $v \subset \tau$ at scale $\delta$. We cover $a+v^{\prime}$ with a family $\left\{T_{\ell}\right\}$ of pairwise disjoint translates of $\tau^{\prime}$ with centres at $\left(x_{\ell}, t_{\ell}\right)$. We can do this in such a way that

$$
\bigcup_{\ell} T_{\ell} \subset a+2 v^{\prime}
$$

As $\Phi_{\tau, \mathbb{V}_{m}, \tau_{m+1}}^{q}$ is essentially constant on $T_{\ell}$, we have

$$
\begin{aligned}
\int_{a+v^{\prime}} \Psi_{v}^{q} & \leq \sum_{\ell} \int_{T_{\ell}} \Psi_{\tau_{\mathbb{V}_{m}, \tau_{m+1}}^{q} \max _{\tau, \mathbb{V}_{m}, \tau_{m+1}}}^{q} \\
& \lesssim \sum_{\ell} \max _{\mathbb{V}_{m}, \tau_{m+1}} \Phi_{\tau, \mathbb{V}_{m}, \tau_{m+1}}^{q}\left(x_{\ell}, t_{\ell}\right)\left|T_{\ell}\right| f_{T_{\ell}} \Psi_{\tau}^{q} .
\end{aligned}
$$

Then, by the induction hypothesis (5.8), we see that

$$
\begin{aligned}
\int_{a+v^{\prime}} \Psi_{v}^{q} & \leq\left|\tau^{\prime}\right|^{\varepsilon} \sum_{\ell} \max _{\mathbb{V}_{m}, \tau_{m+1}} \Phi_{\tau, \mathbb{V}_{m}, \tau_{m+1}}^{q}\left(x_{\ell}, t_{\ell}\right)\left|T_{\ell}\right| \\
& \lesssim\left|\tau^{\prime}\right|^{\varepsilon} \int_{\cup_{\ell} T_{\ell}} \max _{\mathbb{V}_{m}, \tau_{m+1}} \Phi_{\tau, \mathbb{V}_{m}, \tau_{m+1}}^{q} .
\end{aligned}
$$

We are now in a similar position as in the case $l(\tau)=1$. We cover $\bigcup_{\ell} T_{\ell}$ with a family $\left\{T_{j}\right\}$ of disjoint translates of $K_{m+1} \tau^{\prime}$. As the angle between $Y_{v}$ and $Y_{\tau}$ is bounded by $\delta$, elementary trigonometry tells us that we can do this so that

$$
\bigcup_{j} T_{j} \subset a+4 v^{\prime} .
$$


Thus, by Remark 5.1 and Property 5.1,

$$
\begin{aligned}
\int_{a+v^{\prime}} \Psi_{v}^{q} & \leq\left|\tau^{\prime}\right|^{\varepsilon} \sum_{j}\left|T_{j}\right|_{\mathbb{V}_{m}, \tau_{m+1}} f_{T_{j}} \Phi_{\tau, \mathbb{V}_{m}, \tau_{m+1}}^{q} \\
& \lesssim\left|\tau^{\prime}\right|^{\varepsilon} K_{m+1}^{2 \varepsilon} \sum_{j}\left|T_{j}\right| \lesssim\left|\tau^{\prime}\right|^{\varepsilon} K_{m+1}^{2 \varepsilon}\left|v^{\prime}\right| \leq\left|v^{\prime}\right|^{1+\varepsilon}
\end{aligned}
$$

where in the final inequality we used that $\left|v^{\prime}\right|^{1+\varepsilon}=\left|\tau^{\prime}\right|^{\varepsilon} K_{m+1}^{(d+1) \varepsilon}\left|v^{\prime}\right|$, and so the proof is complete.

Returning to the decomposition (5.7), we stop the iteration at the biggest value of $N$ such that $K_{2}^{N} K_{d}<R^{\lambda}$, where $\lambda>0$, so that

$$
\begin{aligned}
\left|T_{S} g\right| \lesssim & R^{\varepsilon} \sum_{R^{-\lambda}<\delta \leq 1} \max _{E_{\delta}}\left(\sum_{\tau \in E_{\delta}} \Psi_{\tau}^{2}\left(\max _{\tau_{1}, \ldots, \tau_{d} \subset \tau} \prod_{k=1}^{d}\left|T_{\tau_{k}} g\right|^{\frac{1}{d}} * \zeta_{\tau_{k}^{\prime}}\right)^{2}\right)^{1 / 2} \\
& +\sum_{R^{-\lambda}<\delta \leq R^{-\lambda+\varepsilon}} \max _{E_{\delta}}\left(\sum_{\tau \in E_{\delta}} \Psi_{\tau}^{2}\left(\left|T_{\tau} g\right| * \zeta_{\tau^{\prime}}\right)^{2}\right)^{1 / 2} \\
& +\sum_{R^{-\lambda}<\delta \leq 1} \max _{E_{\delta}}\left(\sum_{\tau \in E_{\delta}} \Psi_{\tau}^{2}\right)^{1 / 2} R^{-1 / \varepsilon}\|g\|_{L^{2}} .
\end{aligned}
$$

This is what we call the Bourgain-Guth decomposition [11, pp. 1259]. Note that as $\left|\tau^{\prime}\right|<R^{(d+1) \lambda}$, we have

$$
f_{a+\tau^{\prime}} \Psi_{\tau}^{\frac{2(d-1)}{d-2}} \lesssim R^{(d+1) \lambda \varepsilon}, \quad a \in \mathbb{R}^{d}
$$

Later we will dispose of the sets $E_{\delta}$ and take the inner sums in $\tau$ over the full partition of $S$. The outer sum (over the scales at which the partition is taken) has less than $\lambda \mathfrak{c}(\varepsilon)$ terms in it, where $\mathfrak{c}$ is the constant from the Bennett-Carbery-Tao extension estimate. The inequality recalls the way in which the Whitney decomposition can be used to take advantage of bilinear estimates, stopping at a scale for which easy estimates are available. The big difference between this and the Whitney decomposition are the functions $\Psi_{\tau}$, which have reasonably nice properties, but will prove to be something of a hindrance. Indeed, the easy estimates for the linear terms are no longer so good that we can ignore them completely.

\section{Proof of Theorem 1.5}

After the usual duality argument, we will use the Bourgain-Guth decomposition of the previous section in order to bound the extension operator. The first term on the right-hand side of (5.9) will of course be estimated using the Bennett-CarberyTao multilinear estimate from Section 4. The second term on the right-hand side of (5.9) will be estimated using a variant of Sjölin's argument [44], first taking advantage of the fact that the restricted extension operator is essentially constant on tubes of width $R^{\lambda}$ and length $R^{2 \lambda}$. The first estimate worsens as $\lambda$ increases whereas the second estimate improves. The argument is completed by optimising in $\lambda$ so that the two bounds are the same.

Indeed, recalling that by duality the desired estimate (1.2) is equivalent to

$$
\left\|(f d \sigma)^{\vee}(R \cdot)\right\|_{L^{1}(d \mu)} \lesssim R^{-\beta / 2} \sqrt{c_{\alpha}(\mu)\|\mu\|}\|f\|_{L^{2}\left(\mathbb{S}^{d-1}\right)},
$$

by Hölder's inequality, it will suffice to prove

$$
\left\|(f d \sigma)^{\vee}(R \cdot)\right\|_{L^{2}(d \mu)} \lesssim R^{-\beta / 2} \sqrt{c_{\alpha}(\mu)}\|f\|_{L^{2}\left(\mathbb{S}^{d-1}\right)}
$$


for all $\beta$ satisfying

$$
\beta<\alpha-1+\frac{(d-\alpha)^{2}}{(d-1)(2 d-\alpha-1)} .
$$

Defining the measure $\mu_{R}$ by

$$
\int \psi(x) d \mu_{R}(x):=\int \psi(x) R^{\alpha} d \mu(x / R):=R^{\alpha} \int \psi(R x) d \mu(x),
$$

it is clear that $c_{\alpha}\left(\mu_{R}\right)=c_{\alpha}(\mu)$, so that $(6.1)$ is equivalent to

$$
\left\|(f d \sigma)^{\vee}\right\|_{L^{2}\left(d \mu_{R}\right)} \lesssim R^{\frac{\alpha-\beta}{2}} \sqrt{c_{\alpha}(\mu)}\|f\|_{L^{2}\left(\mathbb{S}^{d-1}\right)} .
$$

By a finite splitting, the triangle inequality and the rotational invariance of the inequality (which holds uniformly for all $\alpha$-dimensional measures $\mu_{R}$ ) we can suppose that $\sigma$ is supported on $S=\{(\xi, \phi(\xi)):|\xi| \leq 1 / 2\}$, where $\phi(\xi)=\sqrt{1-|\xi|^{2}}$. Defining

we can write

$$
g(\xi):=\frac{1}{(2 \pi)^{d / 2}} \frac{f(\xi, \phi(\xi))}{\sqrt{1-|\xi|^{2}}}
$$

$$
(f d \sigma)^{\vee}(x, t)=\int_{|\xi| \leq 1 / 2} g(\xi) e^{i x \cdot \xi+i t \phi(\xi)} d \xi
$$

so we see that $(6.2)$ is equivalent to

$$
\left\|T_{S} g\right\|_{L^{2}\left(d \mu_{R}\right)} \lesssim R^{\frac{\alpha-\beta}{2}} \sqrt{c_{\alpha}(\mu)}\|g\|_{L^{2}\left(\mathbb{R}^{d-1}\right)} .
$$

For this we will use the Bourgain-Guth decomposition with $\lambda=\frac{d-\alpha}{2 d-\alpha-1}$;

$$
\begin{aligned}
\left|T_{S} g\right| \lesssim & R^{\varepsilon} \sum_{R^{-\lambda} \leq \delta \leq 1}\left(\sum_{\tau \sim \delta}\left(\Psi_{\tau_{1}, \ldots, \tau_{d} \subset \tau} \prod_{k=1}^{d}\left|T_{\tau_{k}} g\right|^{\frac{1}{d}} * \zeta_{\tau_{k}^{\prime}}\right)^{2}\right)^{1 / 2} \\
& +\sum_{R^{-\lambda} \leq \delta \leq R^{-\lambda+\varepsilon}}\left(\sum_{\tau \sim \delta}\left(\Psi_{\tau}\left|T_{\tau} g\right| * \zeta_{\tau^{\prime}}\right)^{2}\right)^{1 / 2} \\
& +\sum_{R^{-\lambda} \leq \delta \leq 1}\left(\sum_{\tau \sim \delta} \Psi_{\tau}^{2}\right)^{1 / 2} R^{-1 / \varepsilon}\|g\|_{L^{2}\left(\mathbb{R}^{d-1}\right)}
\end{aligned}
$$

which follows from estimate (5.9) by summing in $\tau$ over the full partition of $S$ at scale $\delta$ instead of over the restricted subsets $E_{\delta}$.

Recalling that there are less that $\lambda \mathfrak{c}(\varepsilon)<\mathfrak{c}(\varepsilon)$ terms in each of the $\delta$-sums, by the triangle inequality, we need only prove estimates which are uniform in $\delta$. Writing $g_{\tau}:=g \chi_{\tau}$, if we could prove

$$
\left\|\Psi_{\tau}\left|T_{\tau} g\right| * \zeta_{\tau^{\prime}}\right\|_{L^{2}\left(d \mu_{R}\right)} \lesssim \sqrt{c_{\alpha}(\mu)} R^{\frac{\alpha}{2}-\frac{\alpha-1}{2}-\frac{\lambda(d-\alpha)}{2(d-1)}+d \varepsilon}\left\|g_{\tau}\right\|_{2},
$$

uniformly for $\tau$ at scale $\delta$ with $R^{-\lambda} \leq \delta \leq R^{-\lambda+\varepsilon}$, then using orthogonality, we could bound the middle term on the right-hand side of (6.4). Similarly, replacing the $\max _{\tau_{1}, \ldots, \tau_{d} \subset \tau}$ with an $\ell^{2}$-norm, and using the fact that there are no more than $R^{\varepsilon}$ choices in such a sum, in order to treat the first term it will suffice to prove

$$
\left\|\Psi_{\tau} \prod_{k=1}^{d}\left|T_{\tau_{k}} g\right|^{\frac{1}{d}} * \zeta_{\tau_{k}^{\prime}}\right\|_{L^{2}\left(d \mu_{R}\right)} \lesssim \sqrt{c_{\alpha}(\mu)} R^{\frac{\alpha}{2}-\frac{\alpha-1}{2}-\frac{\lambda(d-\alpha)}{2(d-1)}+d \varepsilon}\left\|g_{\tau}\right\|_{2},
$$

uniformly for $\tau$ at scale $\delta$ with $R^{-\lambda} \leq \delta \leq 1$ and uniformly for choices of transversal caps $\tau_{1}, \ldots, \tau_{d} \subset \tau$. In fact we will only prove this for $\alpha>1$ however we can safely ignore the other cases as Mattila already proved the sharp bound for $\beta_{d}$ for low 
dimensions [35]. Finally, in order to deal with the remainder term, by taking $\varepsilon$ sufficiently small, it will suffice to prove that

$$
\left\|\Psi_{\tau}\right\|_{L^{2}\left(d \mu_{R}\right)} \lesssim \sqrt{c_{\alpha}(\mu)} R^{d / 2+\lambda}
$$

uniformly for $\tau$ at scale $\delta$ with $R^{-\lambda} \leq \delta \leq 1$. Taking for granted the proofs of (6.5), (6.6) and (6.7), which we will present in the forthcoming lemmas, starting with the easier (6.7), this completes the proof of Theorem 1.5.

Lemma 6.1. Let $0<\varepsilon<\frac{1}{4 d}$. Then, for all caps $\tau \sim \delta$ with $R^{-\lambda} \leq \delta \leq 1$,

$$
\left\|\Psi_{\tau}\right\|_{L^{2}\left(d \mu_{R}\right)} \lesssim \sqrt{c_{\alpha}(\mu)} R^{d / 2+\lambda}
$$

Proof. Writing $q=\frac{2(d-1)}{d-2}$, we prepare to use the property (5.10). First of all, as $\Psi_{\tau}$ is essentially constant at scale one, we know that

$$
\begin{aligned}
\int_{B_{j}}\left|\Psi_{\tau}(x)\right|^{q} d \mu_{R}(x) & \leq \mu_{R}\left(B_{j}\right) \sup _{x \in B_{j}}\left|\Psi_{\tau}(x)\right|^{q} \\
& \leq c_{\alpha}\left(\mu_{R}\right) \sup _{x \in B_{j}}\left|\Psi_{\tau}(x)\right|^{q} \lesssim c_{\alpha}\left(\mu_{R}\right) \int_{B_{j}}\left|\Psi_{\tau}(x)\right|^{q} d x
\end{aligned}
$$

whenever $B_{j}$ is a ball of diameter less than one. Thus, we can bound

$$
\begin{aligned}
\left\|\Psi_{\tau}\right\|_{L^{2}\left(d \mu_{R}\right)} & \lesssim \mu_{R}\left(B_{R}\right)^{\frac{1}{2}-\frac{1}{q}}\left\|\Psi_{\tau}\right\|_{L^{q}\left(d \mu_{R}\right)} \\
& \lesssim c_{\alpha}\left(\mu_{R}\right)^{\frac{1}{2}-\frac{1}{q}} R^{\alpha\left(\frac{1}{2}-\frac{1}{q}\right)} c_{\alpha}\left(\mu_{R}\right)^{\frac{1}{q}}\left\|\Psi_{\tau}\right\|_{L^{q}\left(B_{R}\right)} \\
& =\sqrt{c_{\alpha}(\mu)} R^{\alpha\left(\frac{1}{2}-\frac{1}{q}\right)}\left\|\Psi_{\tau}\right\|_{L^{q}\left(B_{R}\right)}
\end{aligned}
$$

Covering $B_{R}$ with a family $\left\{T_{j}\right\}$ of translates of $\tau^{\prime}$ with disjoint interiors, cuboids of dimension $\delta^{-1} \times \cdots \times \delta^{-1} \times \delta^{-2}$, we can then bound this as

$$
\begin{aligned}
\left\|\Psi_{\tau}\right\|_{L^{2}\left(d \mu_{R}\right)} & \lesssim \sqrt{c_{\alpha}(\mu)} R^{\alpha\left(\frac{1}{2}-\frac{1}{q}\right)}\left(\sum_{j}\left\|\Psi_{\tau}\right\|_{L^{q}\left(T_{j}\right)}^{q}\right)^{1 / q} \\
& \lesssim \sqrt{c_{\alpha}(\mu)} R^{\alpha\left(\frac{1}{2}-\frac{1}{q}\right)}\left(\sum_{j}\left|T_{j} \| \tau^{\prime}\right|^{\varepsilon}\right)^{1 / q} \\
& \lesssim \sqrt{c_{\alpha}(\mu)} R^{\frac{d}{2}} \delta^{-\frac{(d+1) \varepsilon}{q}}
\end{aligned}
$$

where the second inequality is by Proposition 5.6. For the range of $\delta$ under consideration, this is easily enough to give the stated bound.

Lemma 6.2. Let $0<\varepsilon<\frac{1}{4 d}$. Then, for all caps $\tau \sim \delta$ with $R^{-\lambda} \leq \delta \leq R^{-\lambda+\varepsilon}$,

$$
\left\|\Psi_{\tau}\left|T_{\tau} g\right| * \zeta_{\tau^{\prime}}\right\|_{L^{2}\left(d \mu_{R}\right)} \lesssim \sqrt{c_{\alpha}(\mu)} R^{\frac{1}{2}-\frac{\lambda(d-\alpha)}{2(d-1)}+d \varepsilon}\left\|g_{\tau}\right\|_{2}
$$

Proof. Again we cover $B_{R}$ by a family $\left\{T_{j}\right\}$ of translations of $\tau^{\prime}$ with disjoint interiors. Setting $G_{\tau}:=\left|T_{\tau} g\right| * \zeta_{\tau^{\prime}}$, and denoting the measure $d \mu_{R}$ restricted to $T_{j}$ by $d \mu_{R}^{j}$, we can write

$$
\left\|\Psi_{\tau} G_{\tau}\right\|_{L^{2}\left(d \mu_{R}\right)}=\left(\sum_{j}\left\|\Psi_{\tau} G_{\tau}\right\|_{L^{2}\left(d \mu_{R}^{j}\right)}^{2}\right)^{1 / 2} .
$$

As in the previous lemma, we use that $\Psi_{\tau}$ is essentially constant at scale one, so

$$
\begin{aligned}
\left\|\Psi_{\tau}\right\|_{L^{2}\left(d \mu_{R}^{j}\right)} & \lesssim \mu_{R}\left(T_{j}\right)^{\frac{1}{2}-\frac{1}{q}}\left\|\Psi_{\tau}\right\|_{L^{q}\left(d \mu_{R}^{j}\right)} \\
& \lesssim \mu_{R}\left(T_{j}\right)^{\frac{1}{2}-\frac{1}{q}} c_{\alpha}\left(\mu_{R}\right)^{\frac{1}{q}}\left\|\Psi_{\tau}\right\|_{L^{q}\left(T_{j}\right)} \\
& \lesssim c_{\alpha}(\mu)^{\frac{1}{q}} \mu_{R}\left(T_{j}\right)^{\frac{1}{2}-\frac{1}{q}} R^{\frac{d+1}{2 q} \varepsilon}\left|T_{j}\right|^{\frac{1}{q}}
\end{aligned}
$$


where the final inequality is by the property (5.10). Using this and the fact that $G_{\tau}$ is essentially constant on $T_{j}$,

$$
\begin{aligned}
\left\|\Psi_{\tau} G_{\tau}\right\|_{L^{2}\left(d \mu_{R}^{j}\right)} & \lesssim c_{\alpha}(\mu)^{\frac{1}{q}} \mu_{R}\left(T_{j}\right)^{\frac{1}{2}-\frac{1}{q}} R^{\frac{d+1}{2 q} \varepsilon}\left|T_{j}\right|^{\frac{1}{q}}\left\|G_{\tau}\right\|_{L^{\infty}\left(T_{j}\right)} \\
& \lesssim c_{\alpha}(\mu)^{\frac{1}{q}} \mu_{R}\left(T_{j}\right)^{\frac{1}{2}-\frac{1}{q}} R^{\frac{d+1}{2 q} \varepsilon}\left|T_{j}\right|^{\frac{1}{q}}\left|T_{j}\right|^{-\frac{1}{2}}\left\|G_{\tau}\right\|_{L^{2}\left(T_{j}\right)} .
\end{aligned}
$$

Plugging this into (8.5), we obtain

$$
\begin{aligned}
\left\|\Psi_{\tau} G_{\tau}\right\|_{L^{2}\left(d \mu_{R}\right)} & \lesssim c_{\alpha}(\mu)^{\frac{1}{q}} \mu_{R}\left(T_{j}\right)^{\frac{1}{2}-\frac{1}{q}} R^{\frac{d+1}{2 q} \varepsilon}\left|T_{j}\right|^{\frac{1}{q}}\left|T_{j}\right|^{-\frac{1}{2}}\left(\sum_{j}\left\|G_{\tau}\right\|_{L^{2}\left(T_{j}\right)}^{2}\right)^{1 / 2} \\
& \lesssim \sqrt{c_{\alpha}(\mu)} R^{\frac{d+1}{2 q} \varepsilon} \delta^{(d-\alpha)\left(\frac{1}{2}-\frac{1}{q}\right)}\left(\sum_{j}\left\|G_{\tau}\right\|_{L^{2}\left(T_{j}\right)}^{2}\right)^{1 / 2} \\
& \lesssim \sqrt{c_{\alpha}(\mu)} R^{\frac{d+1}{2 q} \varepsilon^{\frac{d-\alpha}{2(d-1)}}\left\|G_{\tau}\right\|_{L^{2}\left(B_{R}\right)}}
\end{aligned}
$$

where in the second inequality we use $\mu_{R}\left(T_{j}\right) \lesssim c_{\alpha}(\mu) \delta^{-(\alpha+1)}$ which follows by covering the $T_{j}$ by $\delta^{-1}$ balls of radius $\delta^{-1}$.

On the other hand, by Minkowski's integral inequality, we can bound

$$
\begin{aligned}
\left\|G_{\tau}\right\|_{L^{2}\left(B_{R}\right)}=\left\|\left|T_{\tau} g\right| * \zeta_{\tau^{\prime}}\right\|_{L^{2}\left(B_{R}\right)} & \leq \int\left\|T_{\tau} g(\cdot-y)\right\|_{L^{2}\left(B_{R}\right)} \zeta_{\tau^{\prime}}(y) d y \\
& \leq \int\|\| g_{y}^{\vee}\left\|_{L^{2}\left(\mathbb{R}^{d-1}\right)}\right\|_{L^{2}(|t| \leq R)} \zeta_{\tau^{\prime}}(y) d y,
\end{aligned}
$$

where

$$
g_{y}(\xi):=g(\xi) \chi_{\tau}(\xi) e^{-i \pi(y) \cdot \xi+i\left(t-t_{y}\right) \phi(\xi)}, \quad t_{y}:=y-\pi(y) .
$$

Here $\pi$ is the orthogonal projection onto $\mathbb{R}^{d-1}$. Then by Plancherel's theorem, the fact that $\left\|g_{y}\right\|_{2}=\left\|g_{\tau}\right\|_{2}$, and the fact that the integral of $\zeta_{\tau^{\prime}}$ is bounded, we obtain

$$
\left\|G_{\tau}\right\|_{L^{2}\left(B_{R}\right)} \lesssim R^{1 / 2}\left\|g_{\tau}\right\|_{2}
$$

Plugging this into (6.10), we see that

$$
\left\|\Psi_{\tau}\left|T_{\tau} g\right| * \zeta_{\tau^{\prime}}\right\|_{L^{2}\left(d \mu_{R}\right)} \lesssim \sqrt{c_{\alpha}(\mu)} R^{\frac{1}{2}+\frac{d+2}{2 q} \varepsilon} \delta^{\frac{d-\alpha}{2(d-1)}}\left\|g_{\tau}\right\|_{2},
$$

which, with $R^{-\lambda} \leq \delta \leq R^{-\lambda+\varepsilon}$, yields the desired uniform estimate.

Lemma 6.3. Let $0<\varepsilon<\frac{1}{4 d}$ and $\alpha>1$ and $\lambda=\frac{d-\alpha}{2 d-\alpha-1}$. Then, for all caps $\tau \sim \delta$ with $R^{-\lambda} \leq \delta \leq 1$ and all d-transversal caps $\tau_{1}, \ldots \tau_{d} \sim \delta / K_{d}$ contained in $\tau$,

$$
\left\|\Psi_{\tau} \prod_{k=1}^{d}\left|T_{\tau_{k}} g\right|^{\frac{1}{d}} * \zeta_{\tau_{k}^{\prime}}\right\|_{L^{2}\left(d \mu_{R}\right)} \lesssim \sqrt{c_{\alpha}(\mu)} R^{\frac{1}{2}-\frac{\lambda(d-\alpha)}{2(d-1)}+d \varepsilon}\left\|g_{\tau}\right\|_{2} .
$$

Proof. Setting $G_{\tau}:=\prod_{k=1}^{d}\left|T_{\tau_{k}} g\right|^{\frac{1}{d}} * \zeta_{\tau_{k}^{\prime}}$, we will prove that

$$
\left\|\Psi_{\tau} G_{\tau}\right\|_{L^{2}\left(d \mu_{R}\right)} \lesssim \sqrt{c_{\alpha}(\mu)} R^{d \varepsilon} R^{\frac{\alpha}{2 d}} \delta^{\frac{d-\alpha}{2 d(d-1)}-\frac{d-1}{2 d}}\left\|g_{\tau}\right\|_{2},
$$

which on can calculate gives the required bound for $\delta \geq R^{-\frac{d-\alpha}{2 d-\alpha-1}}$.. By Hölder's inequality with $p=\frac{2 d}{d-1}$, we first note that

$$
\begin{aligned}
\left\|\Psi_{\tau} G_{\tau}\right\|_{L^{2}\left(d \mu_{R}\right)} & \lesssim c_{\alpha}(\mu)^{\frac{1}{2}-\frac{1}{p}} R^{\alpha\left(\frac{1}{2}-\frac{1}{p}\right)}\left\|\Psi_{\tau} G_{\tau}\right\|_{L^{p}\left(d \mu_{R}\right)} \\
& =c_{\alpha}(\mu)^{\frac{1}{2}-\frac{1}{p}} R^{\frac{\alpha}{2 d}}\left(\sum_{j}\left\|\Psi_{\tau} G_{\tau}\right\|_{L^{p}\left(d \mu_{R}^{j}\right)}^{p}\right)^{1 / p}
\end{aligned}
$$


where $d \mu_{R}^{j}$ denotes the measure $d \mu_{R}$ a member of the cover of $B_{R}$ by translates of $\tau^{\prime}$. Using that $\Psi_{\tau}$ is essentially constant at scale one,

$$
\begin{aligned}
\left\|\Psi_{\tau}\right\|_{L^{p}\left(d \mu_{R}^{j}\right)} & \lesssim \mu\left(T_{j}\right)^{\frac{1}{p}-\frac{1}{q}}\left\|\Psi_{\tau}\right\|_{L^{q}\left(d \mu_{R}^{j}\right)} \\
& \lesssim \mu\left(T_{j}\right)^{\frac{1}{p}-\frac{1}{q}} c_{\alpha}(\mu)^{\frac{1}{q}}\left\|\Psi_{\tau}\right\|_{L^{q}\left(T_{j}\right)} \\
& \lesssim \mu\left(T_{j}\right)^{\frac{1}{p}-\frac{1}{q}} c_{\alpha}(\mu)^{\frac{1}{q}}\left|T_{j}\right|^{\frac{1}{q}} R^{\frac{d+1}{2 q} \varepsilon},
\end{aligned}
$$

where the final inequality is by the property (5.10). As $\tau^{\prime} \subset \tau_{k}^{\prime}$ we still have that that $G_{\tau}$ is essentially constant on $T_{j}$, so that

$$
\begin{aligned}
\left\|\Psi_{\tau} G_{\tau}\right\|_{L^{p}\left(d \mu_{R}^{j}\right)} & \lesssim \mu\left(T_{j}\right)^{\frac{1}{p}-\frac{1}{q}} c_{\alpha}(\mu)^{\frac{1}{q}} \mid T_{j} \frac{1}{q}^{\frac{1}{q}} R^{\frac{d+1}{2 q} \varepsilon}\left\|G_{\tau}\right\|_{L^{\infty}\left(T_{j}\right)} \\
& \lesssim \mu\left(T_{j}\right)^{\frac{1}{p}-\frac{1}{q}} c_{\alpha}(\mu)^{\frac{1}{q}} R^{\frac{d+1}{4} \varepsilon} \mid T_{j} j^{\frac{1}{q}-\frac{1}{p}}\left\|G_{\tau}\right\|_{L^{p}\left(T_{j}\right)} \\
& \lesssim c_{\alpha}(\mu)^{\frac{1}{p}} R^{\frac{d+1}{4} \varepsilon} \delta^{(d-\alpha)\left(\frac{1}{p}-\frac{1}{q}\right)}\left\|G_{\tau}\right\|_{L^{p}\left(T_{j}\right)} \\
& =c_{\alpha}(\mu)^{\frac{1}{p}} R^{\frac{d+1}{4} \varepsilon} \delta^{\frac{d-\alpha}{2 d(d-1)}\left\|G_{\tau}\right\|_{L^{p}\left(T_{j}\right)}}
\end{aligned}
$$

Plugging this into (6.14), we obtain

$$
\left\|\Psi_{\tau} G_{\tau}\right\|_{L^{2}\left(d \mu_{R}\right)} \lesssim \sqrt{c_{\alpha}(\mu)} R^{\frac{d+1}{4} \varepsilon} R^{\frac{\alpha}{2 d}} \delta^{\frac{d-\alpha}{2 d(d-1)}}\left\|G_{\tau}\right\|_{L^{p}\left(B_{R}\right)} .
$$

In order to bound $\left\|G_{\tau}\right\|_{L^{p}\left(B_{R}\right)}$, we write

$$
\begin{aligned}
G_{\tau}(x, t) & =\prod_{k=1}^{d} \int\left|T_{\tau_{k}} g\right|^{\frac{1}{d}}\left((x, t)-y_{k}\right) \zeta_{\tau_{k}^{\prime}}\left(y_{k}\right) d y_{k} \\
& =\prod_{k=1}^{d} \int\left|T_{\tau_{k}} g_{y_{k}}\right|^{\frac{1}{d}}(x, t) \zeta_{\tau_{k}^{\prime}}\left(y_{k}\right) d y_{k},
\end{aligned}
$$

where this time

$$
g_{y_{k}}:=g \chi_{\tau_{k}} e^{-i \pi\left(y_{k}\right) \cdot \xi-i t_{k} \phi(\xi)}, \quad t_{k}:=y_{k}-\pi\left(y_{k}\right) .
$$

Then, by Minkowski's integral inequality, it will suffice to bound

$$
\int\left\|\prod_{k=1}^{d}\left|T_{\tau_{k}} g_{y_{k}}\right|^{\frac{1}{d}}\right\|_{L^{p}\left(B_{R}\right)} \prod_{k=1}^{d} \zeta_{\tau_{k}^{\prime}}\left(y_{k}\right) d y_{1} \ldots d y_{d}
$$

Again $\left\|g_{y_{k}}\right\|_{2}=\left\|g_{\tau_{k}}\right\|_{2}$, and so it remains to prove the multilinear extension estimate

$$
\left\|\prod_{k=1}^{d}\left|T_{\tau_{k}} g\right|^{\frac{1}{d}}\right\|_{L^{p}\left(B_{R}\right)} \lesssim R^{2 \varepsilon} \delta^{-\frac{d-1}{2 d}}\left\|g_{\tau}\right\|_{2} .
$$

We recall that $\tau_{k}$ are transversal caps at scale $\delta / K_{d}$ and so a direct application of Theorem 4.1 would give us the inequality with the constant $\mathfrak{c}\left(\delta^{d} K_{d}^{-d}\right)$. We do not know how large this is, however we have chosen the scales so that at least we know that $\mathfrak{c}\left(K_{d}^{-d}\right) \leq R^{\varepsilon^{2}}$. Thus, using the fact the caps $\tau_{k}$ are contained in $\tau$ at scale $\delta$, we can modulate and scale in order to get back into this situation.

Denoting by $\xi_{0}$ the center of $\pi(\tau)=Q$ we let $\widetilde{Q}_{k}$ be the scaled versions of $Q_{k}$ which are first translated by $-\xi_{0}$. Indeed, introducing new variables,

$$
\left(x^{\prime}, t^{\prime}\right)=\left(\delta x, \delta^{2} t\right), \quad \xi-\xi_{0}=\delta \xi^{\prime},
$$

and writing

$$
f\left(\xi^{\prime}\right):=\delta^{\frac{d-1}{2}} g\left(\xi_{0}+\delta \xi^{\prime}\right),
$$

so that $\|f\|_{2}=\|g\|_{2}$, it is trivial to calculate that

$$
\left|T_{\tau_{k}} g(x, t)\right|=\delta^{\frac{d-1}{2}}\left|\widetilde{T}_{\widetilde{\tau}_{k}} f\left(x^{\prime}+\delta^{-1} \nabla \phi\left(\xi_{0}\right) t^{\prime}, t^{\prime}\right)\right|,
$$


where

$$
\widetilde{T}_{\widetilde{\tau}_{k}} f(x, t):=\int_{\widetilde{Q}_{k}} e^{i x \cdot \xi+i t S_{\xi_{0}, \delta} \phi(\xi)} f(\xi) d \xi
$$

and the scaled phase is given by

$$
S_{\xi_{0}, \delta} \phi\left(\xi^{\prime}\right)=\delta^{-2}\left(\phi\left(\xi_{0}+\delta \xi^{\prime}\right)-\delta \nabla \phi\left(\xi_{0}\right) \cdot \xi^{\prime}-\phi\left(\xi_{0}\right)\right) .
$$

The $d$-transversal caps $\widetilde{\tau}_{k}$ satisfying $\pi\left(\widetilde{\tau}_{k}\right)=\widetilde{Q}_{k}$ are now at scale $1 / K_{d}$. Writing

$$
\prod_{k=1}^{d}\left|T_{\tau_{k}} g\right|^{\frac{1}{d}}(x, t)=\delta^{\frac{d-1}{2}} \prod_{k=1}^{d}\left|\widetilde{T}_{\widetilde{\tau}_{k}}\right|^{\frac{1}{d}}\left(x^{\prime}+\delta^{-1} \nabla \phi\left(\xi_{0}\right) t^{\prime}, t^{\prime}\right),
$$

we see that the left-hand side of (6.15) is bounded by

$$
\begin{aligned}
& \delta^{\frac{d-1}{2}-\frac{d+1}{p}}\left(\left.\left.\int_{\left|t^{\prime}\right| \leq \delta^{2} R} \int_{\left|x^{\prime}\right| \leq \delta R}\left|\prod_{k=1}^{d}\right| \widetilde{T}_{\widetilde{\tau}_{k}} f\right|^{\frac{1}{d}}\left(x^{\prime}+\delta^{-1} \nabla \phi\left(\xi_{0}\right) t^{\prime}, t^{\prime}\right)\right|^{p} d x^{\prime} d t^{\prime}\right)^{1 / p} \\
\leq & \delta^{-\frac{d-1}{2 d}}\left\|\prod_{k=1}^{d}\left|\widetilde{T}_{\widetilde{\tau}_{k}} f\right|^{\frac{1}{d}}\right\|_{L^{p}\left(\left[-\delta^{2} R, \delta^{2} R\right] \times B_{2 \delta R}\right)},
\end{aligned}
$$

Here, we change variables $x=x^{\prime}+\delta^{-1} \nabla \phi\left(\xi_{0}\right) t^{\prime}$ and use that $\delta^{-1} \nabla \phi\left(\xi_{0}\right) t^{\prime}$ is bounded above by $\delta R$ so that the oblique tube can be covered by the fatter cylinder. Now, by Proposition 4.3,

$$
\left\|\prod_{k=1}^{d}\left|\widetilde{T}_{\widetilde{\tau}_{k}} f\right|^{\frac{1}{d}}\right\|_{L^{p}\left(\mathbb{R}^{d-1} \times\left[-\delta^{2} R, \delta^{2} R\right]\right)} \lesssim \mathfrak{c}\left(K_{d}^{-d}\right)\left(\delta^{2} R\right)^{\varepsilon}\|f\|_{2} \leq R^{2 \varepsilon}\|f\|_{2},
$$

using the original choice of scales and so altogether we get (6.15), which completes the proof.

The conjectured $m$-linear extension estimates [4, Conjecture 4], with $m \leq d-1$, combined with the arguments of this section, would yield

$$
\beta_{d}(\alpha) \geq \min \left\{\alpha-1+\frac{(d-\alpha)(d+m-2 \alpha)}{2(m-1)(d+m-\alpha-1)}, \alpha-\frac{2 \alpha}{d+m}\right\},
$$

whenever $3 \leq m \leq d-1$. Comparing the second term in the minimum with the bound of Theorem 1.5, it is clear that this is not an improvement for larger $\alpha$. However, by taking $m=d / 2+1$ (assuming that $d$ is even), this would improve our bound and Erdoğan's in a neighbourhood of $\alpha=d / 2+2 / 3$. It would not be sufficient to improve the state-of-the-art for Falconer's conjecture however.

Given that $m$-linear estimates necessarily have worse integrability properties than the $d$-linear estimates of Section 4 , it is not obvious that anything can be gained by lowering the degree of multinearity. The reason that such estimates can be effective is that the decomposition of Bourgain and Guth improves if we take the initial dichotomy at a lower level of multilinearity. The improvement manifests itself in the fact that the functions $\Psi_{\tau}$ have better integrability properties and so we pay less when we remove them. This kind of thing was first observed by Temur in the context of the linear restriction problem [49]. Here, the reduced integrability in the estimates leads to the estimate (6.13) having a worse dependency on $R$ (this produces the second term in the minimum), however the improved properties of $\Psi_{\tau}$ lead to both (6.13) and (6.11) having a better dependency on $\delta$, and together they would yield (6.17) after choosing the limiting scale $\lambda$ in an optimal fashion. 


\section{Proof of Proposition 1.6}

In order to avoid repetition in the following section, we consider $m \geq 1$, however it will suffice to consider $m=1$ here. If $v_{0}$ and $v_{1}$ are in the Schwartz class then the solution $v$ to the wave equation with this initial data can be written as

$$
\begin{aligned}
v(\cdot, t) & =\cos (t \sqrt{-\Delta}) v_{0}+\frac{\sin (t \sqrt{-\Delta})}{\sqrt{-\Delta}} v_{1} \\
& =e^{i t \sqrt{-\Delta}} f_{+}+e^{-i t \sqrt{-\Delta}} f_{-} .
\end{aligned}
$$

Here $f_{+}=\frac{1}{2}\left(v_{0}-i I_{1} * v_{1}\right)$ and $f_{-}=\frac{1}{2}\left(v_{0}+i I_{1} * v_{1}\right)$, where $I_{1}$ is the Riesz kernel, and

$$
e^{i t(-\Delta)^{m / 2}} f(x):=\frac{1}{(2 \pi)^{d / 2}} \int_{\mathbb{R}^{d}} \widehat{f}(\xi) e^{i x \cdot \xi+i t|\xi|^{m}} d \xi .
$$

For data in $\dot{H}^{s} \times \dot{H}^{s-1}$, both $f_{+}$and $f_{-}$belong to $\dot{H}^{s}$, however this integral does not necessarily exist in the sense of Lebesgue for $s \leq n / 2$. Instead we define $v(x, t)$ to be the pointwise limit

$$
v(x, t):=\lim _{N \rightarrow \infty} S_{t}^{N, 1} f_{+}(x)+S_{-t}^{N, 1} f_{-}(x),
$$

whenever the limit exists, where

$$
S_{t}^{N, m} f:=\int_{\mathbb{R}^{d}} \psi\left(\frac{|\xi|}{N}\right) \widehat{f}(\xi) e^{i x \cdot \xi+i t|\xi|^{m}} d \xi
$$

and $\psi$ is a positive Schwartz function that equals $(2 \pi)^{-d / 2}$ at the origin. This coincides almost everywhere with the classical solution defined via the $L^{2}$-limit.

Writing $\left\|I_{s} * f\right\|_{\dot{H}^{s}}:=\|f\|_{2}$, we know that $f_{+}, f_{-}$and the limit (7.1) are welldefined with respect to fractal measures provided that $\alpha>d-2 s$ due to the inequalities

$$
\begin{gathered}
\left\|I_{s} * f\right\|_{L^{1}(d \mu)} \lesssim \sqrt{c_{\alpha}(\mu)\|\mu\|}\|f\|_{2}, \\
\left\|\sup _{N>1}\left|S_{t}^{N, m} I_{s} * f\right|\right\|_{L^{1}(d \mu)} \lesssim \sqrt{c_{\alpha}(\mu)\|\mu\|}\|f\|_{2} ;
\end{gathered}
$$

see for example [2], [6] or [37, Chapter 17]. Then by standard arguments (see for example Appendix B of [6]) and an application of Frostman's lemma (see for example [37, Theorem 2.7]), the implication

$$
\beta_{d}(\alpha)>d-2 s \quad \Rightarrow \quad \gamma_{d}(s) \leq \alpha
$$

can be deduced from from the following lemma.

Lemma 7.1. Let $m \geq 1, d \geq 2$ and $0<s<d / 2$. Then

$$
\left\|\sup _{t \in \mathbb{R}} \sup _{N \geq 1}\left|S_{t}^{N, m} I_{s} * f\right|\right\|_{L^{1}(d \mu)} \lesssim \sqrt{c_{\alpha}(\mu)\|\mu\|}\|f\|_{2}
$$

whenever $f \in L^{2}\left(\mathbb{R}^{d}\right)$, $\mu$ is an $\alpha$-dimensional measure and $s>\frac{d-\beta_{d}(\alpha)}{2}$.

Proof. First of all we remark that the maximal function is Borel measurable by comparing with the maximum function with time restricted to the rationals; see [37, Lemma 17.7]. Then, using polar coordinates we write

$$
\begin{aligned}
\left|S_{t}^{N, m} I_{s} * f(x)\right| & =\left.\left|\int_{\mathbb{R}^{d}} \psi\left(N^{-1}|\xi|\right)\right| \xi\right|^{-s} \widehat{f}(\xi) e^{i\left(x \cdot \xi+t|\xi|^{m}\right)} d \xi \mid \\
& =\left|\int_{0}^{\infty} \psi\left(N^{-1} R\right) R^{d-1-s} e^{i t R^{m}} \int_{\mathbb{S}^{d-1}} \widehat{f}(R \omega) e^{i R x \cdot \omega} d \sigma(\omega) d R\right| \\
& \lesssim \int_{0}^{\infty} R^{d-1-s}\left|\int_{\mathbb{S}^{d-1}} \widehat{f}(R \omega) e^{i R x \cdot \omega} d \sigma(\omega)\right| d R
\end{aligned}
$$


so that, by Fubini's theorem,

$$
\left\|\sup _{t \in \mathbb{R}} \sup _{N \geq 1}\left|S_{t}^{N} I_{s} * f\right|\right\|_{L^{1}(d \mu)} \lesssim \int_{0}^{\infty} R^{d-1-s}\left\|(\widehat{f}(R \cdot) d \sigma)^{\vee}(R \cdot)\right\|_{L^{1}(d \mu)} d R .
$$

Noting that, even when $R$ is small, we have

$$
\|\widehat{\mu}(R \cdot)\|_{L^{2}\left(\mathbb{S}^{d-1}\right)}^{2} \lesssim\|\mu\|^{2} \lesssim c_{\alpha}(\mu)\|\mu\|,
$$

the inequality (1.2) implies by duality that

$$
\left\|(\widehat{f}(R \cdot) d \sigma)^{\vee}(R \cdot)\right\|_{L^{1}(d \mu)} \lesssim \sqrt{c_{\alpha}(\mu)\|\mu\|}(1+R)^{-\beta / 2}\|\widehat{f}(R \cdot)\|_{L^{2}\left(\mathbb{S}^{d-1}\right)} .
$$

for all $\beta<\beta_{d}(\alpha)$, so that (7.2) is bounded by

$$
\lesssim \sqrt{c_{\alpha}(\mu)\|\mu\|} \int_{0}^{\infty} \frac{R^{d-1-s}}{(1+R)^{\beta / 2}}\|\widehat{f}(R \cdot)\|_{L^{2}\left(\mathbb{S}^{d-1}\right)} d R .
$$

Finally, by an application of the Cauchy-Schwarz inequality, we can continue to estimate as

$$
\begin{aligned}
& \lesssim \sqrt{c_{\alpha}(\mu)\|\mu\|}\left(\int_{0}^{\infty} \frac{R^{d-1-2 s}}{(1+R)^{\beta}} d R\right)^{1 / 2}\left(\int_{0}^{\infty}\|\widehat{f}(R \cdot)\|_{L^{2}\left(\mathbb{S}^{d-1}\right)}^{2} R^{d-1} d R\right)^{1 / 2} \\
& \lesssim \sqrt{c_{\alpha}(\mu)\|\mu\|}\|f\|_{L^{2}\left(\mathbb{R}^{d}\right)}
\end{aligned}
$$

where in the final inequality we choose $\beta$ so that $\beta_{d}(\alpha)>\beta>d-2 s$ as we may.

\section{Proof of Theorem 1.2}

As in the previous section, for data in $H^{s}$ we define the solution to the Schrödinger equation to be

$$
u(x, t):=\lim _{N \rightarrow \infty} S_{-t}^{N, 2} u_{0}(x)
$$

whenever the limit exists. This coincides almost everywhere with the classical solution defined via the $L^{2}$-limit. Then, by standard arguments, an upper bound for $\alpha_{n}(s)$ can be obtained from appropriate maximal inequalities with respect to fractal measures. We summarise this in the following lemma.

Lemma 8.1. [2] Let $\alpha>\alpha_{0} \geq n-2 s$ and suppose that

$$
\left\|\sup _{0<t<1}\left|e^{i t \Delta} u_{0}\right|\right\|_{L^{1}(d \mu)} \lesssim \sqrt{c_{\alpha}(\mu)\|\mu\|}\left\|u_{0}\right\|_{H^{s}\left(\mathbb{R}^{n}\right)}
$$

whenever $u_{0}$ is in the Schwartz class and $\mu$ is an $\alpha$-dimensional. Then $\alpha_{n}(s) \leq \alpha_{0}$.

Proof. First we use the argument at the beginning of the proof of Proposition 3.2 in [2] to conclude that (8.1) implies the maximal estimate

$$
\left\|\sup _{0<t<1} \sup _{N>1}\left|S_{-t}^{N, 2} u_{0}\right|\right\|_{L^{1}(d \mu)} \lesssim \sqrt{c_{\alpha}(\mu)\|\mu\|}\left\|u_{0}\right\|_{H^{s+\varepsilon}\left(\mathbb{R}^{n}\right)}
$$

whenever $u_{0} \in H^{s+\varepsilon}$ for all $\varepsilon>0$. Then we use the density argument that invokes Frostman's lemma in the Appendix B of [6] or [37, Chapter 17] to conclude.

Thus it remains to prove a priori maximal estimates that hold uniformly with respect to compactly supported fractal measures. Indeed it remains to prove the following theorem.

Theorem 8.2. Let $n \geq 1$ and

$$
s> \begin{cases}\frac{n-\alpha}{2}+\frac{n}{2(n+1)}, & \text { if } \quad 0 \leq \alpha \leq n-1+\frac{2}{n+1}, \\ (n-\alpha+1)\left(\frac{1}{2}-\frac{1}{4 n}\right) & \text { if } \quad n-1+\frac{2}{n+1} \leq \alpha \leq n .\end{cases}
$$


Then

$$
\left\|\sup _{0<t<1}\left|e^{i t \Delta} f\right|\right\|_{L^{2}(d \mu)} \lesssim \sqrt{c_{\alpha}(\mu)}\|f\|_{H^{s}\left(\mathbb{R}^{n}\right)}
$$

whenever $f$ is Schwartz and $\mu$ is $\alpha$-dimensional.

Proof. Set $s_{\mathrm{O}}=\max \left\{\frac{n-\alpha}{2}+\frac{n}{2(n+1)},(n-\alpha+1)\left(\frac{1}{2}-\frac{1}{4 n}\right)\right\}$. After noting that

$$
\begin{aligned}
\left\|\sup _{0<t<1}\left|e^{i t \Delta} f\right|\right\|_{L^{2}(d \mu)} & \lesssim \sqrt{c_{\alpha}(\mu)}\left\|\sup _{0<t<1}\left|e^{i t \Delta} f\right|\right\|_{L^{\infty}\left(B_{1}\right)} \\
& \lesssim \sqrt{c_{\alpha}(\mu)}\left|B_{2^{c}(\varepsilon)}\right|^{1 / 2}\|\widehat{f}\|_{2} \\
& \lesssim \sqrt{c_{\alpha}(\mu)}\|f\|_{2}
\end{aligned}
$$

provided supp $\widehat{f} \subset\left\{\xi \in \mathbb{R}^{n}:|\xi| \leq 2^{\mathfrak{c}(\varepsilon)}\right\}$, by a dyadic decomposition in frequency, the inequality (8.2) would follow from

$$
\left\|\sup _{0<t<1}\left|e^{i t \Delta} f\right|\right\|_{L^{2}(d \mu)} \lesssim \sqrt{c_{\alpha}(\mu)} R^{s_{\circ}+\varepsilon}\|f\|_{2}
$$

provided supp $\widehat{f} \subset\{R / 8<|\xi|<R / 2\}$ for all $R>2^{\mathfrak{c}(\varepsilon)}$. For this we make use of temporal localisation lemma due to Lee [33, Lemma 2.3]. In fact we use a version that holds with respect to fractal measures and where the $\varepsilon$-loss in derivatives was avoided (see [34, Lemma 2.1]), so that it will suffice to prove

$$
\left\|\sup _{0<t<1 / R}\left|e^{i t \Delta} f\right|\right\|_{L^{2}(d \mu)} \lesssim \sqrt{c_{\alpha}(\mu)} R^{s_{\circ}+\varepsilon}\|f\|_{2} .
$$

Writing $\widehat{f}_{R}=R^{n} \widehat{f}(R \cdot)$ and scaling, we see that

$$
\left\|\sup _{0<t<1 / R}\left|e^{i t \Delta} f\right|\right\|_{L^{2}(d \mu)}=R^{-\alpha / 2}\left(\int \sup _{0<t<R}\left|e^{i t \Delta} f_{R}\right|^{2}(x) R^{\alpha} d \mu(x / R)\right)^{1 / 2}
$$

so that, by writing $d \mu_{R}(x):=R^{\alpha} d \mu(x / R)$, this is equivalent to

$$
\left\|\sup _{0<t<R}\left|e^{i t \Delta} f\right|\right\|_{L^{2}\left(d \mu_{R}\right)} \lesssim \sqrt{c_{\alpha}(\mu)} R^{\frac{\alpha-n}{2}+s_{\circ}+\varepsilon}\|f\|_{2},
$$

provided supp $\widehat{f} \subset\{\xi: 1 / 8 \leq|\xi| \leq 1 / 2\}$. It is easy to check that $c_{\alpha}\left(\mu_{R}\right)=c_{\alpha}(\mu)$.

Now by taking $\lambda=1 / 2$ in (5.9) we have the pointwise bound

$$
\begin{aligned}
\left.\left|e^{i t \Delta} f\right| \lesssim R_{R^{-1 / 2} \leq \delta \leq 1}^{\varepsilon} \sum_{\tau \sim \delta}\left(\sum_{\tau_{1}, \ldots, \tau_{n+1} \subset \tau} \prod_{k=1}^{n+1}\left|T_{\tau_{k}} \widehat{f}\right|^{\frac{1}{n+1}} * \zeta_{\tau_{k}^{\prime}}\right)^{2}\right)^{1 / 2} \\
+\sum_{R^{-1 / 2} \leq \delta \leq R^{-1 / 2+\varepsilon}}\left(\sum_{\tau \sim \delta}\left(\Psi_{\tau}\left|T_{\tau} \widehat{f}\right| * \zeta_{\tau^{\prime}}\right)^{2}\right)^{1 / 2} \\
+\sum_{R^{-1 / 2} \leq \delta \leq 1}\left(\sum_{\tau \sim \delta} \Psi_{\tau}^{2}\right)^{1 / 2} R^{-1 / \varepsilon}\|\widehat{f}\|_{2} .
\end{aligned}
$$

Recalling that there are a finite number, independent of $R$, of terms in each of the $\delta$-sums, by the triangle inequality, we need only prove estimates which are uniform in $\delta$. Writing $g_{\tau}:=\widehat{f} \chi_{\tau}$, if we could prove

$$
\left\|\sup _{0<t<R} \Psi_{\tau}\left|T_{\tau} g\right| * \zeta_{\tau^{\prime}}\right\|_{L^{2}\left(d \mu_{R}\right)} \lesssim \sqrt{c_{\alpha}(\mu)} R^{\frac{\alpha-n}{2}+s_{\mathrm{o}}+n \varepsilon}\left\|g_{\tau}\right\|_{2}
$$

uniformly for $\tau$ at scale $\delta$ with $R^{-1 / 2} \leq \delta \leq R^{-1 / 2+\varepsilon}$, then using orthogonality, we could bound the middle term on the right-hand side of (8.2). Similarly, replacing 
the $\max _{\tau_{1}, \ldots, \tau_{n+1} \subset \tau}$ with a $\ell^{2}$-norm, and using the fact that there are no more than $R^{\varepsilon}$ choices in such a sum, in order to treat the first term it will suffice to prove

$$
\left\|\sup _{0<t<R} \Psi_{\tau} \prod_{k=1}^{n+1}\left|T_{\tau_{k}} g\right|^{\frac{1}{n+1}} * \zeta_{\tau_{k}^{\prime}}\right\|_{L^{2}\left(d \mu_{R}\right)} \lesssim \sqrt{c_{\alpha}(\mu)} R^{\frac{\alpha-n}{2}+s_{\mathrm{o}}+n \varepsilon}\left\|g_{\tau}\right\|_{2},
$$

uniformly for $\tau$ at scale $\delta$ with $R^{-1 / 2} \leq \delta \leq 1$ and uniformly for choices of transversal caps $\tau_{1}, \ldots, \tau_{n+1} \subset \tau$. Finally, in order to deal with the remainder term, by taking $\varepsilon$ sufficiently small, it will suffice to prove that

$$
\left\|\sup _{0<t<R} \Psi_{\tau}\right\|_{L^{2}\left(d \mu_{R}\right)} \lesssim \sqrt{c_{\alpha}(\mu)} R^{n+1},
$$

uniformly for $\tau$ at scale $\delta$ with $R^{-1 / 2} \leq \delta \leq 1$. Taking for granted the proofs of $(8.2),(8.3)$ and $(8.4)$, which we will present in the forthcoming lemmas, this completes the proof of Theorem 8.2.

From now on, for nested norms, we write $\|f\|_{X Y}:=\|\| f\left\|_{Y}\right\|_{X}$.

Lemma 8.3. Let $0<\varepsilon<\frac{1}{8 n}$. Then, for all caps $\tau \sim \delta$ with $R^{-1 / 2} \leq \delta \leq 1$,

$$
\left\|\Psi_{\tau}\right\|_{L^{2}\left(d \mu_{R}\right) L^{\infty}(0, R)} \lesssim \sqrt{c_{\alpha}(\mu)} R^{n+1} .
$$

Proof. Writing $q=\frac{2 n}{n-1}$, we prepare to apply Proposition 5.6. First of all, as $\Psi_{\tau}$ is essentially constant at scale one, we can bound

$$
\begin{aligned}
\left\|\Psi_{\tau}\right\|_{L^{2}\left(d \mu_{R}\right) L^{\infty}(0, R)} & \lesssim\left\|\Psi_{\tau}\right\|_{L^{2}\left(d \mu_{R}\right) L^{q}(0, R)} \\
& \lesssim \sqrt{c_{\alpha}(\mu)}\left\|\Psi_{\tau}\right\|_{L^{2}\left(B_{R}\right) L^{q}(0, R)} \\
& \lesssim \sqrt{c_{\alpha}(\mu)} R^{n\left(\frac{1}{2}-\frac{1}{q}\right)}\left\|\Psi_{\tau}\right\|_{L^{q}\left(B_{R} \times(0, R)\right)} .
\end{aligned}
$$

Noting that $n\left(\frac{1}{2}-\frac{1}{q}\right)=\frac{1}{2}$, and covering $B_{R} \times(0, R)$ with a family $\left\{T_{j}\right\}$ of translates of $\tau^{\prime}$ with disjoint interiors, we can bound this as

$$
\begin{aligned}
\left\|\Psi_{\tau}\right\|_{L^{2}\left(d \mu_{R}\right) L^{q}(0, R)} & \lesssim \sqrt{c_{\alpha}(\mu)} R^{\frac{1}{2}}\left(\sum_{j}\left\|\Psi_{\tau}\right\|_{L^{q}\left(T_{j}\right)}^{q}\right)^{1 / q} \\
& \lesssim \sqrt{c_{\alpha}(\mu)} R^{\frac{1}{2}}\left(\sum_{j}\left|T_{j} \| \tau^{\prime}\right|^{\varepsilon}\right)^{1 / q} \\
& \lesssim \sqrt{c_{\alpha}(\mu)} R^{\frac{1}{2}} R^{\frac{n+1}{q}} \delta^{-\frac{(n+2) \varepsilon}{q}}
\end{aligned}
$$

where the second inequality is by Proposition 5.6. For the range of $\delta$ under consideration, this is more than enough to give the desired bound.

Lemma 8.4. Let $0<\varepsilon<\frac{1}{8 n}$. Then, for all caps $\tau \sim \delta$ with $R^{-1 / 2} \leq \delta \leq R^{-1 / 2+\varepsilon}$,

$$
\left\|\Psi_{\tau}\left|T_{\tau} g\right| * \zeta_{\tau^{\prime}}\right\|_{L^{2}\left(d \mu_{R}\right) L^{\infty}(0, R)} \lesssim \sqrt{c_{\alpha}(\mu)} R^{\frac{1}{2}-\frac{1}{4 n}-\frac{n-\alpha}{4 n}+n \varepsilon}\left\|g_{\tau}\right\|_{2} .
$$

Proof. We cover $B_{R} \times(0, R)$ by a family $\left\{T_{j k}\right\}$ of translations of $\tau^{\prime}$ with disjoint interiors. Denote by $I_{j}$ the projection orthogonal to time of $T_{j k}$ onto $\mathbb{R}^{n}$. Recall that the sets $T_{j k}$ have dimensions $\delta^{-1} \times \cdots \times \delta^{-1} \times \delta^{-2}$ and, as our functions are frequency supported in the the unit annulus, the sets $\tau^{\prime}$ make an angle greater than $\pi / 8$ with the time axis. Thus the projections $I_{j}$ also have a long side of length a constant multiple of $\delta^{-2}$. 
Set $G_{\tau}:=\left|T_{\tau} g\right| * \zeta_{\tau^{\prime}}$. Denoting by $d \mu_{R}^{j}$ the measure $d \mu_{R}$ restricted to $I_{j}$, by Hölder's inequality

$$
\begin{aligned}
\left\|\Psi_{\tau} G_{\tau}\right\|_{L^{2}\left(d \mu_{R}\right) L^{\infty}(0, R)} & =\left(\sum_{j}\left\|\Psi_{\tau} G_{\tau}\right\|_{L^{2}\left(d \mu_{R}^{j}\right) L^{\infty}(0, R)}^{2}\right)^{1 / 2} \\
& \leq \mu_{R}\left(I_{j}\right)^{\frac{1}{2}-\frac{1}{p}}\left(\sum_{j}\left\|\Psi_{\tau} G_{\tau}\right\|_{L^{p}\left(d \mu_{R}^{j}\right) L^{\infty}(0, R)}^{2}\right)^{1 / 2}
\end{aligned}
$$

Denoting $T_{j k}^{x}=\left\{(y, t) \in T_{j k}: y=x\right\}$, on the other hand we have

$$
\sup _{0<t<R}\left|\Psi_{\tau} G_{\tau}\right|(x, t) \leq\left(\sum_{k}\left\|\Psi_{\tau} G_{\tau}\right\|_{L^{\infty}\left(B_{j k}^{x}\right)}^{p}\right)^{1 / p},
$$

for all $x \in I_{j}$, so that

$$
\begin{aligned}
& \left\|\Psi_{\tau} G_{\tau}\right\|_{L^{2}\left(d \mu_{R}\right) L^{\infty}(0, R)} \\
& \quad \leq \mu_{R}\left(I_{j}\right)^{\frac{1}{2}-\frac{1}{p}}\left(\sum_{j}\left(\sum_{k}\left\|\Psi_{\tau} G_{\tau}\right\|_{L^{p} L^{\infty}\left(T_{j k}, d \mu_{R} d t\right)}^{p}\right)^{2 / p}\right)^{1 / 2} .
\end{aligned}
$$

As in the previous lemma, we use that $\Psi_{\tau}$ is essentially constant at scale one, so that

$$
\begin{aligned}
\left\|\Psi_{\tau}\right\|_{L^{p} L^{\infty}\left(T_{j k}, d \mu_{R} d t\right)} & \lesssim\left\|\Psi_{\tau}\right\|_{L^{p} L^{q}\left(T_{j k}, d \mu_{R} d t\right)} \\
& \leq \mu_{R}\left(I_{j}\right)^{\frac{1}{p}-\frac{1}{q}}\left\|\Psi_{\tau}\right\|_{L^{q}\left(T_{j k}, d \mu_{R} d t\right)} \\
& \lesssim \mu_{R}\left(I_{j}\right)^{\frac{1}{p}-\frac{1}{q}} c_{\alpha}(\mu)^{\frac{1}{q}}\left\|\Psi_{\tau}\right\|_{L^{q}\left(T_{j k}, d x d t\right)} \\
& \lesssim c_{\alpha}(\mu)^{\frac{1}{q}} \mu_{R}\left(I_{j}\right)^{\frac{1}{p}-\frac{1}{q}} R^{\frac{n+2}{2 q} \varepsilon}\left|T_{j k}\right|^{\frac{1}{q}},
\end{aligned}
$$

where the final inequality is by (5.10). Using this and the fact that $G_{\tau}$ is essentially constant on $T_{j k}$,

$$
\begin{aligned}
\left\|\Psi_{\tau} G_{\tau}\right\|_{L^{p} L^{\infty}\left(T_{j k}, d \mu_{R} d t\right)} & \lesssim c_{\alpha}(\mu)^{\frac{1}{q}} \mu_{R}\left(I_{j}\right)^{\frac{1}{p}-\frac{1}{q}} R^{\frac{n+2}{2 q} \varepsilon}\left|T_{j k}\right|^{\frac{1}{q}}\left\|G_{\tau}\right\|_{L^{\infty} L^{\infty}\left(T_{j k}\right)} \\
& \lesssim c_{\alpha}(\mu)^{\frac{1}{q}} \mu_{R}\left(I_{j}\right)^{\frac{1}{p}-\frac{1}{q}} R^{\frac{n+2}{4} \varepsilon}\left|T_{j k}\right|^{\frac{1}{q}} \delta^{\frac{n+1}{2}} \delta^{\frac{1}{p}}\left\|G_{\tau}\right\|_{L^{2} L^{p}\left(T_{j k}\right)} .
\end{aligned}
$$

Plugging this into (8.5), we obtain

$$
\begin{aligned}
&\left\|\Psi_{\tau} G_{\tau}\right\|_{L^{2}\left(d \mu_{R}\right) L^{\infty}(0, R)} \\
& \lesssim c_{\alpha}(\mu)^{\frac{1}{q}} \mu_{R}\left(I_{j}\right)^{\frac{1}{2}-\frac{1}{q}} R^{\frac{n+2}{4} \varepsilon}\left|T_{j k}\right|^{\frac{1}{q}} \delta^{\frac{n+1}{2}} \delta^{\frac{1}{p}}\left(\sum_{j}\left(\sum_{k}\left\|G_{\tau}\right\|_{L^{2} L^{p}\left(T_{j k}\right)}^{p}\right)^{2 / p}\right)^{1 / 2} \\
& \lesssim \sqrt{c_{\alpha}(\mu)} R^{\frac{n+2}{4}} \varepsilon \delta^{-(\alpha+1)\left(\frac{1}{2}-\frac{1}{q}\right)} \delta^{(n+1)\left(\frac{1}{2}-\frac{1}{q}\right)+\frac{1}{p}-\frac{1}{q}}\left(\sum_{j}\left(\sum_{k}\left\|G_{\tau}\right\|_{L^{2} L^{p}\left(T_{j k}\right)}^{p}\right)^{2 / p}\right)^{1 / 2}
\end{aligned}
$$

where in the second inequality we use $\mu_{R}\left(I_{j}\right) \lesssim c_{\alpha}(\mu) \delta^{-(\alpha+1)}$ which follows by covering the $I_{j}$ by $\delta^{-1}$ balls of radius $\delta^{-1}$. Finally, using that $G_{\tau}$ is essentially constant on $T_{j k}$ and $\frac{1}{2}-\frac{1}{q}=\frac{1}{2 n}$, we can sum up to obtain

$$
\left\|\Psi_{\tau} G_{\tau}\right\|_{L^{2}\left(d \mu_{R}\right) L^{\infty}(0, R)} \lesssim \sqrt{c_{\alpha}(\mu)} R^{\frac{n+2}{4} \varepsilon} \delta^{\frac{n-\alpha}{2 n}+\frac{1}{p}-\frac{1}{q}}\left\|G_{\tau}\right\|_{L^{2}\left(B_{R}\right) L^{p}(0, R)}
$$


In fact we have only performed this argument for general $p$ to facilitate the proof of the following lemma. Here we set $p=2$ and so it remains to bound

$$
\begin{aligned}
\left\|T_{\tau} g \mid * \zeta_{\tau^{\prime}}\right\|_{L^{2}\left(B_{R}\right) L^{2}(0, R)} & \leq \int\left\|T_{\tau} g(\cdot-y)\right\|_{L^{2}(0, R) L^{2}\left(\mathbb{R}^{n}\right)} \zeta_{\tau^{\prime}}(y) d y \\
& \leq \int\left\|g_{\tau}\right\|_{L^{2}(0, R) L^{2}\left(\mathbb{R}^{n}\right)} \zeta_{\tau^{\prime}}(y) d y \\
& \lesssim\left\|g_{\tau}\right\|_{L^{2}(0, R) L^{2}\left(\mathbb{R}^{n}\right)}=R^{1 / 2}\left\|g_{\tau}\right\|_{L^{2}\left(\mathbb{R}^{n}\right)},
\end{aligned}
$$

by Fubini, Minkowski's integral inequality and Plancherel. Plugging this into the previous estimate, we see that

$$
\left\|\Psi_{\tau}\left|T_{\tau} g\right| * \zeta_{\tau^{\prime}}\right\|_{L^{2}\left(d \mu_{R}\right) L^{\infty}(0, R)} \lesssim \sqrt{c_{\alpha}(\mu)} R^{\frac{1}{2}+\frac{n+2}{4} \varepsilon} \delta^{\frac{n-\alpha}{2 n}+\frac{1}{2 n}}\left\|g_{\tau}\right\|_{2}
$$

which, with $R^{-1 / 2} \leq \delta \leq R^{-1 / 2+\varepsilon}$, yields the desired uniform estimate.

Lemma 8.5. Let $0<\varepsilon<\frac{1}{8 n}$. Then, for all caps $\tau \sim \delta$ with $R^{-1 / 2} \leq \delta \leq 1$ and all $(n+1)$-transversal caps $\tau_{1}, \ldots \tau_{n+1} \sim \delta / K_{n+1}$ contained in $\tau$,

$$
\begin{aligned}
&\left\|\Psi_{\tau} \prod_{k=1}^{n+1}\left|T_{\tau_{k}} g\right|^{\frac{1}{n+1}} * \zeta_{\tau_{k}^{\prime}}\right\|_{L^{2}\left(d \mu_{R}\right) L^{\infty}(0, R)} \\
& \lesssim \sqrt{c_{\alpha}(\mu)} R^{n \varepsilon} \max \left(R^{\frac{1}{2}-\frac{1}{4 n}-\frac{n-\alpha}{4 n}}, R^{\frac{n}{2(n+1)}}\right)\left\|g_{\tau}\right\|_{2} .
\end{aligned}
$$

Proof. As before we set $G_{\tau}:=\prod_{k=1}^{n+1}\left|T_{\tau_{k}} g\right|^{\frac{1}{n+1}} * \zeta_{\tau_{k}^{\prime}}$, and this time we will prove

$$
\left\|\Psi_{\tau} G_{\tau}\right\|_{L^{2}\left(d \mu_{R}\right) L^{\infty}(0, R)} \lesssim \sqrt{c_{\alpha}(\mu)} R^{\frac{n}{2(n+1)}+n \varepsilon} \delta^{\frac{n-\alpha}{2 n}+\frac{1}{2 n}-\frac{1}{n+1}}\left\|g_{\tau}\right\|_{2},
$$

which yields the desired estimate uniform in the range $R^{-1 / 2} \leq \delta \leq 1$. Covering $B_{R} \times(0, R)$ by translations of $\tau^{\prime}$, as $\tau^{\prime} \subset \tau_{k}^{\prime}$ we still have that $G_{\tau}$ is essentially constant at this scale. Repeating the previous argument, this time with $p:=\frac{2(n+1)}{n}$, by (8.6) we have

$$
\left\|\Psi_{\tau} G_{\tau}\right\|_{L^{2}\left(d \mu_{R}\right) L^{\infty}(0, R)} \lesssim \sqrt{c_{\alpha}(\mu)} R^{\frac{n+2}{4} \varepsilon} \delta^{\frac{n-\alpha}{2 n}+\frac{1}{p}-\frac{1}{q}}\left\|G_{\tau}\right\|_{L^{2}\left(B_{R}\right) L^{p}(0, R)},
$$

and so it remains to bound $\left\|G_{\tau}\right\|_{L^{2}\left(B_{R}\right) L^{p}(0, R)}$. By Minkowski's integral inequality, it will suffice to treat

$$
\int\left\|\prod_{k=1}^{n+1}\left|T_{\tau_{k}} g_{y_{k}}\right|^{\frac{1}{n+1}}\right\|_{L^{2}\left(B_{R}\right) L^{p}(0, R)} \prod_{k=1}^{n+1} \zeta_{\tau_{k}^{\prime}}\left(y_{k}\right) d y_{1} \ldots d y_{n+1}
$$

where

$$
g_{y_{k}}:=g \chi_{\tau_{k}} e^{-i \pi\left(y_{k}\right) \cdot \xi+t_{k}|\xi|^{2}}, \quad t_{k}:=y_{k}-\pi\left(y_{k}\right) .
$$

Noting that $\frac{1}{p}-\frac{1}{q}=\frac{1}{2 n}-\frac{1}{2(n+1)}$ and $\left\|g_{y_{k}}\right\|_{2}=\left\|g_{\tau_{k}}\right\|_{2}$, it remains to prove

$$
\left\|\prod_{k=1}^{n+1}\left|T_{\tau_{k}} g\right|^{\frac{1}{n+1}}\right\|_{L^{2}\left(B_{R}\right) L^{p}(0, R)} \lesssim R^{\frac{n}{2(n+1)}+\varepsilon} \delta^{-\frac{1}{2(n+1)}}\|g\|_{2}
$$

By scaling as in the proof of Lemma 6.3 (see (6.16) for the definition), this would follow from

$$
\left\|\prod_{k=1}^{n+1}\left|\widetilde{T}_{\widetilde{\tau}_{k}} f\left(x^{\prime}-2 \delta^{-1} \xi_{0} t^{\prime}, t^{\prime}\right)\right|^{\frac{1}{n+1}}\right\|_{L^{2}\left(B_{\delta R}\right) L^{p}\left(0, \delta^{2} R\right)} \lesssim R^{\frac{n}{2(n+1)}+\varepsilon} \delta^{\frac{2}{p}-\frac{1}{2(n+1)}}\|f\|_{2} .
$$

By a rotation we can suppose that $\xi_{0}$ is parallel to $x_{n}$, so by an application of Hölder's inequality, and making the change of variables $x=x^{\prime}-2 \delta^{-1} \xi_{0} t^{\prime}$, it would 
suffice to prove

$$
\left\|\prod_{k=1}^{n+1}\left|\widetilde{T}_{\widetilde{\tau}_{k}} f\right|^{\frac{1}{n+1}}\right\|_{L^{2}\left(B_{\delta R}\right) L_{x_{n}, t}^{p}(-2 \delta R, 2 \delta R) \times\left(0, \delta^{2} R\right)} \lesssim R^{\frac{n-1}{2(n+1)}+\varepsilon} \delta^{\frac{3}{p}-\frac{1}{2}-\frac{1}{2(n+1)}}\|f\|_{2} .
$$

Now partitioning $\mathbb{R}^{n-1}$ into cubes $\Omega$ of side length $\delta^{2} R$, and applying Hölder's inequality, the left-hand side is bounded by

$$
\left(\delta^{2} R\right)^{(n-1)\left(\frac{1}{2}-\frac{1}{p}\right)}\left(\sum_{\Omega}\left\|\prod_{k=1}^{n+1}\left|\widetilde{T}_{\widetilde{\tau}_{k}} f\right|^{\frac{1}{n+1}}\right\|_{L^{p}(\Omega) L_{x_{n}, t}^{p}(-2 \delta R, 2 \delta R) \times\left(0, \delta^{2} R\right)}^{2}\right)^{1 / 2} .
$$

Noting that

$$
2(n-1)\left(\frac{1}{2}-\frac{1}{p}\right)=\frac{n-1}{n+1}=\frac{3}{p}-\frac{1}{2}-\frac{1}{2(n+1)},
$$

the proof is completed by an application of Proposition 4.3.

\section{Appendix}

The following lemma is well-known; see for example [49, pp. 1024].

Lemma 8.6. Let $\widehat{\psi}=\widehat{\psi}_{\mathrm{o}} * \widehat{\psi}_{\mathrm{o}}$ be a smooth radially symmetric cut-off function supported in $B(0, d) \subset \mathbb{R}^{d}$ and equal to one on $B(0, \sqrt{d})$ and consider the scaled version $\phi_{\tau^{\prime}}$ adapted to $\tau^{\prime}$. Then, for all $m \geq 1$,

$$
|F(x, t)| \lesssim\left(|F|^{\frac{1}{m}} *\left|\psi_{\tau^{\prime}}\right|^{\frac{1}{m}}(x, t)\right)^{m}
$$

provided supp $\widehat{F} \subset \tau \subset \mathbb{R}^{d}$.

Proof. As usual we set $m^{\prime}:=m /(m-1)$. Letting

$$
\eta(x, t):=\psi_{\tau^{\prime}}(x, t) e^{i x \cdot \xi_{\mathrm{o}}+i t \phi\left(\xi_{\mathrm{o}}\right)},
$$

where $\left(\xi_{\mathrm{o}}, \phi\left(\xi_{\mathrm{o}}\right)\right)$ is the centre of $\tau$, we note that

$$
|\eta|^{\frac{1}{m}}=\left|\tau^{\prime}\right|^{\frac{1}{m^{\prime}}}\left|\psi_{\tau^{\prime}}\right|^{\frac{1}{m}} \text {. }
$$

By the self reproducing formula $F=F * \eta$

$$
\begin{aligned}
|F(x, t)| & \leq \int|F((x, t)-y) \eta(y)| d y \\
& =\int|F((x, t)-y) \eta(y)|^{\frac{1}{m}}|F((x, t)-y) \eta(y)|^{\frac{1}{m^{\prime}}} d y \\
& \leq\|F((x, t)-\cdot) \eta\|_{L^{\infty}}^{\frac{1}{m^{\prime}}} \int|F((x, t)-y) \eta(y)|^{\frac{1}{m}} d y \\
& \lesssim\left|\tau^{\prime}\right|^{-\frac{1}{m^{\prime}}}\|F((x, t)-\cdot) \eta\|_{L^{1}}^{\frac{1}{m^{\prime}}} \int|F((x, t)-y) \eta(y)|^{\frac{1}{m}} d y
\end{aligned}
$$

where in the last inequality we have used Bernstein's inequality. Hence by dividing by $\|F((x, t)-\cdot) \eta\|_{L^{1}}^{\frac{1}{m^{\prime}}}$, we see that

$$
\begin{aligned}
\left(\int|F((x, t)-y) \eta(y)| d y\right)^{\frac{1}{m}} & \lesssim\left|\tau^{\prime}\right|^{-\frac{1}{m^{\prime}}}\left(\int|F((x, t)-y) \eta(y)|^{\frac{1}{m}} d y\right) \\
& =\left(\left.\int\left|F((x, t)-y)^{\frac{1}{m}}\right| \psi_{\tau^{\prime}}(y)\right|^{\frac{1}{m}} d y\right),
\end{aligned}
$$

where in the final identity we have used (8.9). Then (8.7) follows using (8.10). 
Lemma 8.7. Let $0<\delta \leq 1$ and let $K>\left(K^{\prime}\right)^{2}>1$. Let $\Lambda_{1}, \Lambda_{2} \in S O(d)$ be such that $\Lambda_{1} \Lambda_{2}^{-1}$ is a rotation by an angle less than $\delta$. Then if $F: \mathbb{R}^{d} \rightarrow \mathbb{R}_{+}$is essentially constant on translates of $\Lambda_{1}^{-1}(T)$ where

$$
T:=\left[-\frac{K}{\delta}, \frac{K}{\delta}\right] \times \cdots \times\left[-\frac{K}{\delta}, \frac{K}{\delta}\right] \times\left[-\frac{K}{\delta^{2}}, \frac{K}{\delta^{2}}\right],
$$

and

or

$$
\zeta(x, t) \lesssim \frac{\delta^{d+1}}{\left(K^{\prime}\right)^{d}}\left(1+\left|\frac{\delta x}{K^{\prime}}\right|^{2}+\left|\frac{\delta^{2} t}{K^{\prime}}\right|^{2}\right)^{-N}
$$

for some $N \geq d$, then

$$
\zeta(x, t) \lesssim \frac{\delta^{d+1}}{\left(K^{\prime}\right)^{d+1}}\left(1+\left|\frac{\delta x}{K^{\prime}}\right|^{2}+\left|\frac{\delta^{2} t}{\left(K^{\prime}\right)^{2}}\right|^{2}\right)^{-N}
$$

$$
F * \zeta\left(\Lambda_{2}(\cdot)\right)\left(x_{1}, t_{1}\right) \lesssim_{L} F\left(x_{2}, t_{2}\right)+\|F\|_{L^{\infty}}\left(\frac{K^{\prime}}{K}\right)^{N}
$$

whenever $\left(x_{1}, t_{1}\right)-\left(x_{2}, t_{2}\right) \in \Lambda_{1}^{-1}(T)$.

Proof. If $\zeta$ takes the second form, then by a change of variables,

$$
\begin{aligned}
\int_{\mathbb{R}^{d} / T} \zeta(x, t) d x d t & \lesssim \int_{\mathbb{R}^{d} /\left[-K / K^{\prime}, K / K^{\prime}\right]^{d-1} \times\left[-K /\left(K^{\prime}\right)^{2}, K /\left(K^{\prime}\right)^{2}\right]}|(x, t)|^{-2 N} d x d t \\
& \lesssim \int_{K / K^{\prime}}^{\infty} \rho^{-2 N+d-1} d \rho \lesssim_{N}\left(\frac{K^{\prime}}{K}\right)^{2 N-d},
\end{aligned}
$$

and the same is true if $\zeta$ takes the first form. Then note that

$$
\begin{aligned}
& \int F\left(\left(x_{1}, t_{1}\right)-y\right) \zeta\left(\Lambda_{2}(y)\right) d y=\int F\left(\left(x_{1}, t_{1}\right)-\Lambda_{2}^{-1} y\right) \zeta(y) d y \\
= & \int_{T} F\left(\left(x_{1}, t_{1}\right)-\Lambda_{2}^{-1} y\right) \zeta(y) d y+\int_{\mathbb{R}^{d} / T} F\left(\left(x_{1}, t_{1}\right)-\Lambda_{2}^{-1} y\right) \zeta(y) d y=: I+I I .
\end{aligned}
$$

By trigonometry and the essentially constant assumption, we have

$$
\left.F\left(\left(x_{1}, t_{1}\right)-\Lambda_{2}^{-1} y\right)\right|_{y \in \Lambda_{1}^{-1} T} \lesssim F\left(x_{2}, t_{2}\right),
$$

whenever $\left(x_{1}, t_{1}\right)-\left(x_{2}, t_{2}\right) \in \Lambda_{1}^{-1} T$ so that $I \lesssim F\left(x_{2}, t_{2}\right)$. On the other hand, we have that

$$
I I \leq\|F\|_{L^{\infty}} \int_{\mathbb{R}^{d} / T} \zeta(y) d y \lesssim\|F\|_{L^{\infty}}\left(\frac{K^{\prime}}{K}\right)^{2 N-d} .
$$

from before, and so the desired estimate follows by adding the two bounds.

\section{REFERENCES}

[1] K. Atkinson and W. Han, Spherical Harmonics and Approximations to the Unit Sphere: An Introduction, Springer-Verlag, Berlin, 2012.

[2] J. A. Barceló, J. Bennett, A. Carbery and K. M. Rogers, On the dimension of divergence sets of dispersive equations, Math. Ann. 349 (2011), no. 3, 599-622.

[3] J. A. Barceló, J. Bennett, A. Carbery, A. Ruiz, and M. C. Vilela, Some special solutions of the Schrödinger equation, Indiana Univ. Math. J. 56 (2007), no. 4, 1581-1593.

[4] J. Bennett, Aspects of multilinear harmonic analysis related to transversality, in Harmonic analysis and partial differential equations, 1-28, Contemp. Math. 612, Amer. Math. Soc., Providence, RI.

[5] J. Bennett, A. Carbery and T. Tao, On the multilinear restriction and Kakeya conjectures, Acta Math. 196 (2006), no. 2, 261-302.

[6] J. Bennett and K. M. Rogers, On the size of divergence sets for the Schrödinger equation with radial data, Indiana Univ. Math. J. 61 (2012), no. 1, 1-13.

[7] J. Bourgain, A remark on Schrödinger operators, Israel J. Math. 77 (1992), no. 1-2, 1-16.

[8] _ Hausdorff dimension and distance sets, Israel J. Math. 87 (1994), no. 1-3, 193-201. 
[9] Some new estimates on oscillatory integrals, in Essays on Fourier analysis in honor of Elias M. Stein (Princeton, NJ, 1991), Princeton Math. Ser. 42 (1995), 83-112.

[10] _ On the Schrödinger maximal function in higher dimension, Tr. Mat. Inst. Steklova 280 (2013), 53-66.

[11] J. Bourgain and L. Guth, Bounds on oscillatory integral operators based on multilinear estimates, Geom. Funct. Anal. 21 (2011), no. 6, 1239-1295.

[12] L. Brandolini, G. Gigante, A. Greenleaf, I. Iosevich, A. Seeger, and G. Travaglini, Average decay estimates for Fourier transforms of measures supported on curves, J. Geom. Anal. 17 (2007), no. 1, 15-40.

[13] L. Brandolini, S. Hofmann, and A. Iosevich, Sharp rate of average decay of the Fourier transform of a bounded set, Geom. Funct. Anal. 13 (2003), 671-680.

[14] A. Carbery, Radial Fourier multipliers and associated maximal functions, in Recent progress in Fourier analysis (El Escorial, 1983), 49-56, North-Holland Math. Stud. 11, North-Holland, Amsterdam.

[15] C.-H. Cho and S. Lee, Dimension of divergence sets for the pointwise convergence of the Schrödinger equation, J. Math. Anal. Appl. 411 (2014), no. 1, 254-260.

[16] A. Carbery and F. Soria, Pointwise Fourier inversion and localisation in $\mathbb{R}^{n}$, J. Fourier Anal. Appl. 3 (1997), Special Issue, 847-858.

[17] L. Carleson, Some analytic problems related to statistical mechanics, in Euclidean harmonic analysis (Proc. Sem., Univ. Maryland, College Park, Md., 1979), 5-45, Lecture Notes in Math. 779, Springer, Berlin.

[18] L. Colzani, G. Gigante and A. Vargas, Localization for Riesz means of Fourier expansions, Trans. Amer. Math. Soc. 366 (2014), no. 12, 6229-6245.

[19] M. Cowling, Pointwise behavior of solutions to Schrödinger equations, in Harmonic analysis (Cortona, 1982), 83-90, Lecture Notes in Math. 992, Springer, Berlin.

[20] B. E. J. Dahlberg and C. E. Kenig, A note on the almost everywhere behavior of solutions to the Schrödinger equation, in Harmonic analysis (Minneapolis, Minn., 1981), 205-209, Lecture Notes in Math. 908, Springer, Berlin.

[21] M. B. Erdoğan, A note on the Fourier transform of fractal measures, Math. Res. Lett. 11 (2004), no. 2-3, 299-313.

[22] A B bilinear Fourier extension theorem and applications to the distance set problem, Int. Math. Res. Not. no. 23 (2005), 1411-1425.

[23] _ On Falconer's distance set conjecture, Rev. Mat. Iberoam. 22 (2006), no. 2, 649-662.

[24] F. Fricker, Einführung in die Gitterpunktlehre, Birkhäuser (1982).

[25] G. Gigante and F. Soria, On the boundedness in $H^{1 / 4}$ of the maximal square function associated with the Schrödinger equation, J. Lond. Math. Soc. (2) 77 (2008), no. 1, 51-68.

[26] L. Grafakos, Classical Fourier analysis, third edition, Graduate Texts in Mathematics, 249, Springer, New York, 2014.

[27] A. Greenleaf, I. Iosevich, B. Liu and E. Palsson, A group theoretic viewpoint on ErdősFalconer problems and the Mattila integral, 31 (2015), 799-810.

[28] S. Ham and S. Lee, Restriction estimates for space curves with respect to general measures, Adv. Math. 254 (2014), 251-279.

[29] S. Hofmann and A. Iosevich, Circular averages and Falconer/Erdös distance conjecture in the plane for random metrics, Proc. Amer. Math. Soc. 133 (2005), no. 1, 133-143

[30] A. Iosevich and E. Liflyand, Decay of the Fourier transform: Analytic and Geometric Aspects, Birkhäuser Mathematics, Basel, 2015.

[31] A. Iosevich and M. Rudnev, On the Mattila integral associated with sign indefinite measures, J. Fourier Anal. Appl. 13 (2007), no. 2, 167-173.

[32] , Distance measures for well-distributed sets, Discrete Comput. Geom. 38 (2007), 61-80.

[33] S. Lee, On pointwise convergence of the solutions to the Schrödinger equations in $\mathbb{R}^{2}$. Int. Math. Res. Not. (2006), 1-21.

[34] S. Lee and K. M. Rogers, The Schrödinger equation along curves and the quantum harmonic oscillator, Adv. Math. 229 (2012), no. 3, 1359-1379

[35] P. Mattila, Spherical averages of Fourier transforms of measures with finite energy; dimension of intersections and distance sets, Mathematika 34 (1987), no. 2, 207-228.

[36] _ Hausdorff dimension, projections, and the Fourier transform, Publ. Mat. 48 (2004), no. $1,3-48$.

[37] _ Fourier analysis and Hausdorff dimension, Cambridge Studies in Advanced Mathematics 150, Cambridge Univ. Press, Cambridge, 2015.

[38] A. Moyua, A. Vargas and L. Vega, Schrödinger maximal function and restriction properties of the Fourier transform, Int. Math. Res. Not. 16 (1996), 793-815. 
[39] , Restriction theorems and maximal operators related to oscillatory integrals in $\mathbb{R}^{3}$, Duke Math. J. 96 (1999), no. 3, 547-574.

[40] E. M. Nikišin, A resonance theorem and series in eigenfunctions of the Laplace operator, Izv. Akad. Nauk SSSR Ser. Mat. 36 (1972), 795-813.

[41] D. M. Oberlin and R. Oberlin, Application of a Fourier restriction theorem to certain families of projections in $\mathbb{R}^{3}$, J. Geom. Anal. 25 (2015), 1476-1491.

[42] P. Sjögren and P. Sjölin, Convergence properties for the time-dependent Schrödinger equation, Ann. Acad. Sci. Fenn. 14 (1989), no. 1, 13-25.

[43] P. Sjölin, Regularity of solutions to the Schrödinger equation, Duke Math. J. 55 (1987), no. 3, 699-715.

[44] _ Estimates of spherical averages of Fourier transforms and dimensions of sets, Mathematika 40 (1993), no. 2, 322-330.

[45] P. Sjölin and F. Soria, Estimates of averages of Fourier transforms with respect to general measures, Proc. Roy. Soc. Edinburgh Sect. A, 133 (2003), 943-950.

[46] E. M. Stein, On limits of sequences of operators, Ann. of Math. (2), 74 (1961), 140-170.

[47] T. Tao, A sharp bilinear restrictions estimate for paraboloids, Geom. Funct. Anal. 13 (2003), no. $6,1359-1384$.

[48] T. Tao and A. Vargas, A bilinear approach to cone multipliers II. Applications, Geom. Funct. Anal. 10 (2000), no. 1, 185-258.

[49] F. Temur, A Fourier restriction estimate for surfaces of positive curvature in $\mathbb{R}^{6}$, Rev. Mat. Iberoam. 30 (2014), no. 3, 1015-1036.

[50] L. Vega, Schrödinger equations: pointwise convergence to the initial data, Proc. Amer. Math. Soc. 102 (1988), no. 4, 874-878.

[51] B. G. Walther, Some $L^{p}\left(L^{\infty}\right)$ - and $L^{2}\left(L^{2}\right)$-estimates for oscillatory Fourier transforms, in Analysis of divergence (Orono, MA, 1997), 213-231, Appl. Numer. Harmon. Anal., Birkhäuser, Boston, MA.

[52] T. H. Wolff, Decay of circular means of Fourier transforms of measures, Int. Math. Res. Not. (1999), 547-567.

[53] D. Žubrinić, Singular sets of Sobolev functions, C. R. Math. Acad. Sci. Paris 334 (2002), no. $7,539-544$.

Department Mathematik und Informatik, Universität Basel, Basel 4051, SwitzerLAND.

Email address: renato.luca@unibas.ch

Instituto de Ciencias Matemáticas CSiC-UAM-UC3M-UCM, Madrid 28049, Spain.

Email address: keith.rogers@icmat.es 